\title{
IMPLICIT PARTIAL DIFFERENTIAL EQUATIONS AND THE CONSTRAINTS OF NON LINEAR ELASTICITY
}

\begin{abstract}
We study a Dirichlet problem associated to some nonlinear partial differential equations under additional constraints that are relevant in non linear elasticity. We also give several examples related to the complex eikonal equation, optimal design, potential wells or nematic elastomers.
\end{abstract}

\section{Bernard Dacorogna}

Département de Mathématiques, EPFL, 1015 Lausanne, Switzerland E-mail address: Bernard.Dacorogna@epfl.ch

\section{Chiara Tanteri}

Département de Mathématiques, EPFL, 1015 Lausanne, Switzerland E-mail address: Chiara.Tanteri@epfl.ch

\section{INTRODUCTION}

We consider here the following Dirichlet problem (as well as some higher order versions of the problem)

$$
\left\{\begin{array}{l}
F_{i}(x, u(x), D u(x))=0, \quad i=1,2, \ldots, I, \text { a.e. } x \in \Omega \\
u=\varphi, \text { on } \partial \Omega
\end{array}\right.
$$

where $\Omega \subset \mathbb{R}^{n}$ is a bounded open set, $u: \Omega \rightarrow \mathbb{R}^{n}$ and therefore $D u \in \mathbb{R}^{n \times n}, F_{i}: \mathbb{R}^{n \times n} \rightarrow \mathbb{R}, i=1, \ldots, I$, are quasiconvex functions and $\varphi$, the boundary datum, is given.

This problem has been intensively studied and we refer to DacorognaMarcellini [6] for a discussion of these implicit equations. We will be interested here in considering the case where we require that the solutions satisfy some constraints that are natural in non linear elasticity. The first one is the non interpenetration of matter that is expressed mathematically by det $D u>0$ and the second one is the incompressibility which reads as det $D u=1$. These two questions were raised in [6] and are discussed in Dacorogna-Marcellini-Tanteri [7] for the first one

Key words and phrases. Rank one convex hull, implicit partial differential equations. 
and in Müller-Sverak [14] for the second one, using a different approach based on the method of convex integration of Gromov.

We will discuss here some theoretical results related to the first (Section 2) and second (Section 3,4) cases and deal with several relevant examples (Section 5 to 10). We will also make some general considerations concerning polyconvex hulls (Section 11) and we will conclude in an Appendix (Section 12) with some well known properties of singular values of matrices.

We now describe six examples that we will investigate here, but we first recall that we respectively denote by $\operatorname{co} E$, Pco $E$, Rco $E$, the convex, polyconvex, rank one convex hull of a given set $E \subset \mathbb{R}^{m \times n}$.

The first example has already been considered when $n=2$ in Dacorogna-Marcellini [6].

Example 1.1 (Complex eikonal equation). The problem has been introduced by Magnanini and Talenti [12] motivated by problems of geometrical optics with diffraction. Given $\Omega \subset \mathbb{R}^{n}$ a bounded open set, $f: \Omega \times \mathbb{R} \times \mathbb{R} \rightarrow \mathbb{R}, f=f(x, u, v)$, a continuous function, we wish to find a complex function $w \in W^{1, \infty}(\Omega ; \mathbb{C})$

$$
w(x)=u(x)+i v(x)
$$

such that

$$
\left\{\begin{array}{l}
\sum_{i=1}^{n} w_{x_{i}}^{2}+f^{2}=0, \quad \text { a.e. in } \Omega \\
w=\varphi, \quad \text { on } \partial \Omega
\end{array}\right.
$$

where $w_{x_{i}}=\partial w / \partial x_{i}$. The problem is then equivalent to

$$
\left\{\begin{array}{l}
|D v|^{2}=|D u|^{2}+f^{2}, \quad \text { a.e. in } \Omega, \\
\langle D v ; D u\rangle=0, \quad \text { a.e. in } \Omega, \\
w=\varphi, \text { on } \partial \Omega .
\end{array}\right.
$$

We will solve, in fact, a more restrictive problem, namely

$$
\left\{\begin{array}{l}
|D v|^{2}=r^{2}+f^{2}, \quad|D u|^{2}=r^{2}, \quad \text { a.e. in } \Omega \\
\langle D v ; D u\rangle=0, \quad \text { a.e. in } \Omega, \\
w=\varphi, \text { on } \partial \Omega,
\end{array}\right.
$$

for an appropriate $r>0$. In algebraic terms (at this point we can consider $f$ to be constant) we have, letting $s=\sqrt{r^{2}+f^{2}}$,

$$
E=\left\{\xi=\left(\begin{array}{c}
a \\
b
\end{array}\right) \in \mathbb{R}^{2 \times n}:|a|=r,|b|=s \text { and }\langle a ; b\rangle=0\right\} .
$$

Letting

$$
A=\left(\begin{array}{ll}
1 / r & 0 \\
0 & 1 / s
\end{array}\right) \in \mathbb{R}^{2 \times 2}
$$


we will prove that

$$
\text { Pco } E=\operatorname{Rco} E=\left\{\xi \in \mathbb{R}^{2 \times n}: \lambda_{1}(A \xi), \lambda_{2}(A \xi) \leq 1\right\}
$$

where $\lambda_{1}(A \xi), \lambda_{2}(A \xi)$ are the singular values of the matrix $A \xi \in \mathbb{R}^{2 \times n}$ (c.f. the Appendix for more details).

The second example is important for optimal design and is related to the method of confocal ellipses of Murat-Tartar [17] and of the results of Dacorogna-Marcellini [4] and [6]. However the existence part will be obtained without the use of the confocal ellipses method, contrary to the one in [4] and [6].

Example 1.2 (Optimal design). Let $\Omega \subset \mathbb{R}^{2}$ be a bounded open set and consider the Dirichlet-Neumann problem

$$
\left\{\begin{array}{l}
\Delta w(x) \in\{0,1\}, \quad \text { a.e. } x \in \Omega, \\
\operatorname{det} D^{2} w(x) \geq 0, \quad \text { a.e. } x \in \Omega, \\
w(x)=\varphi(x), D w(x)=D \varphi(x), \quad x \in \partial \Omega .
\end{array}\right.
$$

The associated algebraic problem is (denoting the set of $2 \times 2$ symmetric matrices by $\mathbb{R}_{s}^{2 \times 2}$ ) when we let

$$
E=\left\{\xi \in \mathbb{R}_{s}^{2 \times 2}: \operatorname{trace} \xi \in\{0,1\}, \operatorname{det} \xi \geq 0\right\},
$$

to find that

$$
\text { Rco } E=\operatorname{co} E=\left\{\xi \in \mathbb{R}_{s}^{2 \times 2}: 0 \leq \operatorname{trace} \xi \leq 1, \operatorname{det} \xi \geq 0\right\} .
$$

We will next consider two more academic examples but that exhibit some interesting features. The first one shows how we can handle some problems depending on singular values under a constraint on the positivity of the determinant.

Example 1.3. Let $\Omega \subset \mathbb{R}^{2}$ be an open set and consider

$$
\left\{\begin{array}{c}
\lambda_{1}(D u)+\lambda_{2}(D u)=1, \text { a.e. in } \Omega \\
\operatorname{det} D u>0, \text { a.e. in } \Omega \\
u(x)=\varphi(x), \quad x \in \partial \Omega .
\end{array}\right.
$$

The associated algebraic problem is : given

$$
E=\left\{\xi \in \mathbb{R}^{2 \times 2}: \lambda_{1}(\xi)+\lambda_{2}(\xi)=1, \quad \operatorname{det} \xi \geq 0\right\}
$$

to prove that

$$
\text { Pco } E=\operatorname{Rco} E=\left\{\xi \in \mathbb{R}^{2 \times 2}: \lambda_{1}(\xi)+\lambda_{2}(\xi) \leq 1, \quad \operatorname{det} \xi \geq 0\right\} .
$$

The fourth problem that we want to discuss is the following second order problem. 
Example 1.4. Let $\Omega \subset \mathbb{R}^{2}$ be a bounded open set and consider the Dirichlet-Neumann problem

$$
\left\{\begin{array}{l}
\left|\frac{\partial^{2} u}{\partial x_{i} \partial x_{j}}\right|=1, \quad \text { a.e. } x \in \Omega, \quad i, j=1,2 \\
u(x)=\varphi(x), D u(x)=D \varphi(x), \quad x \in \partial \Omega .
\end{array}\right.
$$

The algebraic problem is then

$$
E=\left\{\xi=\left(\xi_{i j}\right) \in \mathbb{R}_{s}^{2 \times 2}:\left|\xi_{i j}\right|=1, \quad i, j=1,2\right\}
$$

and we will find that

$$
\operatorname{Pco} E=\operatorname{Rco} E=\left\{\begin{array}{c}
\xi=\left(\xi_{i j}\right) \in \mathbb{R}_{s}^{2 \times 2}:\left|\xi_{i j}\right| \leq 1, \quad i, j=1,2 \\
\left|\xi_{11}-\xi_{22}\right| \leq-\operatorname{det} \xi
\end{array}\right\} .
$$

The last two examples concern the incompressibility constraint. The first one is the problem of two potential wells in two dimensions that was resolved by Müller-Sverak in [13] and by Dacorogna-Marcellini in [5] and [6] for the case $\operatorname{det} A \neq \operatorname{det} B$ and by Müller-Sverak in [14] for the case $\operatorname{det} A=\operatorname{det} B$. In Section 9 we will show that our results also apply to this problem.

Example 1.5 (Potential wells). Let $\Omega \subset \mathbb{R}^{n}$ be open and

$$
E=S O(2) A \cup S O(2) B
$$

with $\operatorname{det} A=\operatorname{det} B>0$. Let

$$
\xi \in \operatorname{int} \operatorname{Rco} E
$$

where int Rco $E$ stands for the interior (relative to the manifold $\operatorname{det} \xi=$ $\operatorname{det} A=\operatorname{det} B$ ) of the rank one convex hull of $E$ (c.f. Section 9 for the characterization of Rco $E)$. Then there exists $u \in W^{1, \infty}\left(\Omega ; \mathbb{R}^{2}\right)$ such that

$$
\left\{\begin{array}{c}
D u(x) \in E, \text { a.e. in } \Omega \\
u(x)=\xi x, \text { on } \partial \Omega .
\end{array}\right.
$$

The last example is related to some recent work of DeSimone-Dolzmann [9] on nematic elastomers; we refer to this article for the description of the physical model.

Example 1.6 (Nematic elastomers). Let $r<1$ (this is called the oblate case while $r>1$ is called the prolate case and it can be handled similarly), let $0 \leq \lambda_{1}(A) \leq \ldots \leq \lambda_{n}(A)$ denote the singular values of a matrix $A \in \mathbb{R}^{n \times n}$ and

$$
E=\left\{A: \lambda_{\nu}(A)=r^{\frac{1}{2 n}}, 1 \leq \nu \leq n-1, \lambda_{n}(A)=r^{\frac{(1-n)}{2 n}}, \operatorname{det} A=1\right\} .
$$


We will prove that

$$
\begin{aligned}
\operatorname{Rco} E & =\left\{A: \prod_{i=\nu}^{n} \lambda_{i}(A) \leq r^{(1-\nu) / 2 n}, 2 \leq \nu \leq n, \operatorname{det} A=1\right\} \\
& =\left\{A: \lambda_{\nu}(A) \in\left[r^{1 / 2 n}, r^{(1-n) / 2 n}\right], 1 \leq \nu \leq n, \operatorname{det} A=1\right\}
\end{aligned}
$$

(this representation formula, under the second form, has been established in [9] when $n=2,3$; actually we will consider below a slightly more general case). Our analytical result is then: given $\xi \in \operatorname{int} \operatorname{Rco} E$ and $\Omega \subset \mathbb{R}^{n}$ an open set, there exists $u \in \varphi+W_{0}^{1, \infty}\left(\Omega ; \mathbb{R}^{n}\right)(\varphi(x)=\xi x)$ such that

$$
\left\{\begin{array}{l}
\lambda_{1}(D u(x))=\ldots=\lambda_{n-1}(D u(x))=r^{1 / 2 n}, \text { a.e. } x \in \Omega \\
\lambda_{n}(D u(x))=r^{(1-n) / 2 n}, \text { a.e. } x \in \Omega \\
\operatorname{det} D u(x)=1, \text { a.e. } x \in \Omega .
\end{array}\right.
$$

\section{INEQUALITY CONSTRAints}

The results of this section are inspired by those of Dacorogna-MarcelliniTanteri [7].

We recall first some notations and definitions introduced in [6].

Notations: (1) Let $N, n, m \geq 1$ be integers. For $u: \mathbb{R}^{n} \rightarrow \mathbb{R}^{m}$ we write

$$
D^{N} u=\left(\frac{\partial^{N} u^{i}}{\partial x_{j_{1}} \ldots \partial x_{j_{N}}}\right)_{1 \leq j_{1}, \ldots, j_{N} \leq n}^{1 \leq i \leq m} \in \mathbb{R}_{s}^{m \times n^{N}} .
$$

(The index $s$ stands here for all the natural symmetries implied by the interchange of the order of differentiation). When $N=1$ we have

$$
\mathbb{R}_{s}^{m \times n}=\mathbb{R}^{m \times n}
$$

while if $m=1$ and $N=2$ we obtain

$$
\mathbb{R}_{s}^{n^{2}}=\mathbb{R}_{s}^{n \times n}
$$

i.e., the usual set of symmetric matrices.

(2) For $u: \Omega \rightarrow \mathbb{R}^{m}$ we let

$$
D^{[N]} u=\left(u, D u, \ldots, D^{N} u\right)
$$

stand for the matrix of all partial derivatives of $u$ up to the order $N$. Note that

$D^{[N]} u \in \mathbb{R}_{s}^{m \times M}=\mathbb{R}^{m} \times \mathbb{R}^{m \times n} \times \mathbb{R}_{s}^{m \times n^{2}} \times \ldots \times \mathbb{R}_{s}^{m \times n^{(N-1)}}$,

where

$$
M=1+n+\ldots+n^{(N-1)}=\frac{n^{N}-1}{n-1} .
$$


Hence

$$
D^{[N]} u=\left(D^{[N-1]} u, D^{N} u\right) \in \mathbb{R}_{s}^{m \times M} \times \mathbb{R}_{s}^{m \times n^{N}} .
$$

We now define the main property, called the relaxation property (c.f. [6]), in order to get existence of solution.

Definition 2.1 (Relaxation property). Let $E, K \subset \mathbb{R}^{n} \times \mathbb{R}_{s}^{m \times M} \times$ $\mathbb{R}_{s}^{m \times n^{N}}$. We say that $K$ has the relaxation property with respect to $E$ if for every bounded open set $\Omega \subset \mathbb{R}^{n}$, for every $u_{\xi}$, a polynomial of degree $N$ with $D^{N} u_{\xi}(x)=\xi$, satisfying

$$
\left(x, D^{[N-1]} u_{\xi}(x), D^{N} u_{\xi}(x)\right) \in \operatorname{int} K,
$$

there exists a sequence $u_{\nu} \in C_{\text {piec }}^{N}\left(\bar{\Omega} ; \mathbb{R}^{m}\right)$ such that

$$
\begin{aligned}
& u_{\nu} \in u_{\xi}+W_{0}^{N, \infty}\left(\Omega ; \mathbb{R}^{m}\right) \\
& u_{\nu} \stackrel{*}{*} u_{\xi} \text { in } W^{N, \infty} \\
& \left(x, D^{[N-1]} u_{\nu}(x), D^{N} u_{\nu}(x)\right) \in E \cup \operatorname{int} K, \text { a.e in } \Omega \\
& \int_{\Omega} \operatorname{dist}\left(\left(x, D^{[N-1]} u_{\nu}(x), D^{N} u_{\nu}(x)\right) ; E\right) d x \rightarrow 0 \text { as } \nu \rightarrow \infty .
\end{aligned}
$$

The following theorem is the main abstract existence theorem.

Theorem 2.2. Let $\Omega \subset \mathbb{R}^{n}$ be open. Let $F_{i}: \Omega \times \mathbb{R}_{s}^{m \times M} \times \mathbb{R}_{s}^{m \times n^{N}} \rightarrow$ $\mathbb{R}, F_{i}=F_{i}(x, s, \xi), i=1,2, \ldots, I$, be continuous with respect to all variables and quasiconvex with respect to the variable $\xi$. Let $E, K \subset$ $\mathbb{R}^{n} \times \mathbb{R}_{s}^{m \times M} \times \mathbb{R}_{s}^{m \times n^{N}}$ be such that

$$
\begin{aligned}
& E=\left\{(x, s, \xi): \begin{array}{l}
F_{i}(x, s, \xi)=0,1 \leq i \leq I_{1} \\
F_{i}(x, s, \xi) \leq 0, I_{1}+1 \leq i \leq I
\end{array}\right\} \\
& K \subset\left\{(x, s, \xi): F_{i}(x, s, \xi) \leq 0,1 \leq i \leq I\right\} .
\end{aligned}
$$

Assume that the set in the right hand side of the inclusion is bounded uniformly for $x \in \Omega$ and whenever $s$ vary on a bounded set of $\mathbb{R}_{s}^{m \times M}$ and that $K$ has the relaxation property with respect to E. Let $\varphi \in$ $C_{\text {piec }}^{N}\left(\bar{\Omega} ; \mathbb{R}^{m}\right)$ such that

$$
\left(x, D^{[N-1]} \varphi(x), D^{N} \varphi(x)\right) \in E \cup \operatorname{int} K \text {, a.e in } \Omega ;
$$

then there exists (a dense set of) $u \in \varphi+W_{0}^{N, \infty}\left(\Omega ; \mathbb{R}^{m}\right)$ such that

$$
\left\{\begin{array}{l}
F_{i}\left(x, D^{[N-1]} u(x), D^{N} u(x)\right)=0, \quad i=1,2, \ldots, I_{1}, \text { a.e. } x \in \Omega \\
F_{i}\left(x, D^{[N-1]} u(x), D^{N} u(x)\right) \leq 0, \quad i=I_{1}+1, \ldots, I, \text { a.e. } x \in \Omega .
\end{array}\right.
$$

Remark 2.3. (1) This result has been proved in Dacorogna-Marcellini [6] (Theorem 6.3) when $I=I_{1}$. With substantially the same proof it can be found in Dacorogna-Marcellini-Tanteri [7].

(2) An interesting case of constraints is when $I=I_{1}+1$ and

$$
F_{I_{1}+1}(\xi)=-\operatorname{det} \xi
$$


(i.e. $\operatorname{det} \xi \geq 0)$. It is actually this constraint that will be used in two of the examples below.

If the set $E$ is given by only one equation the theorem takes a simpler form.

Theorem 2.4. Let $\Omega \subset \mathbb{R}^{n}$ be open. Let $F: \Omega \times \mathbb{R}^{n} \times \mathbb{R}^{n \times n} \rightarrow \mathbb{R}$ be continuous and quasiconvex. Assume that $\left\{\xi \in \mathbb{R}^{n \times n}: F(x, s, \xi) \leq 0\right.$, $\operatorname{det} \xi>0\}$ is bounded in $\mathbb{R}^{n \times n}$ uniformly with respect to $x \in \Omega$ and $s$ in a bounded set of $\mathbb{R}^{n}$. If $\varphi \in C_{\text {piec }}^{1}\left(\bar{\Omega} ; \mathbb{R}^{n}\right)$ is such that

$$
\left\{\begin{array}{c}
F(x, \varphi(x), D \varphi(x)) \leq 0, \text { a.e. } x \in \Omega, \\
\operatorname{det} D \varphi(x)>0, \text { a.e. } x \in \Omega,
\end{array}\right.
$$

then there exists (a dense set of) $u \in \varphi+W_{0}^{1, \infty}\left(\Omega ; \mathbb{R}^{n}\right)$ such that

$$
\left\{\begin{array}{l}
F(x, u(x), D u(x))=0, \text { a.e. } x \in \Omega \\
\operatorname{det} D u(x)>0, \text { a.e. } x \in \Omega .
\end{array}\right.
$$

Remark 2.5. The fact that we can treat strict inequalities follows from the observation that, by hypothesis, we can find $\delta>0$ such that $\operatorname{det} D \varphi>\delta$ since $\varphi \in C_{\text {piec. }}^{1}$. The remaining part of the proof follows from the next theorem.

We have a generalization of the above theorem.

Theorem 2.6. Let $\Omega \subset \mathbb{R}^{n}$ be open. Let $F, \Phi: \Omega \times \mathbb{R}_{s}^{m \times M} \times \mathbb{R}_{s}^{m \times n^{N}} \rightarrow \mathbb{R}$ be continuous and respectively quasiconvex and quasiaffine. Assume that

$$
\left\{\xi \in \mathbb{R}_{s}^{m \times n^{N}}: F(x, s, \xi) \leq 0, \Phi(x, s, \xi) \leq 0\right\}
$$

is bounded in $\mathbb{R}_{s}^{m \times n^{N}}$ uniformly with respect to $x \in \Omega$ and $s$ in a bounded set of $\mathbb{R}_{s}^{m \times M}$. If $\varphi \in C_{\text {piec }}^{N}\left(\bar{\Omega} ; \mathbb{R}^{m}\right)$ is such that

$$
\left\{\begin{array}{l}
F\left(x, D^{[N-1]} \varphi(x), D^{N} \varphi(x)\right) \leq 0, \text { a.e. } x \in \Omega \\
\Phi\left(x, D^{[N-1]} \varphi(x), D^{N} \varphi(x)\right)<0, \text { a.e. } x \in \Omega
\end{array}\right.
$$

then there exists (a dense set of) $u \in \varphi+W_{0}^{N, \infty}\left(\Omega ; \mathbb{R}^{m}\right)$ such that

$$
\left\{\begin{array}{l}
F\left(x, D^{[N-1]} u(x), D^{N} u(x)\right)=0, \text { a.e. } x \in \Omega \\
\Phi\left(x, D^{[N-1]} u(x), D^{N} u(x)\right) \leq 0, \text { a.e. } x \in \Omega .
\end{array}\right.
$$

Proof. We will do the proof when $\varphi$ is affine and when there is no dependence on lower order terms, i.e.

$$
E=\left\{\xi \in \mathbb{R}_{s}^{m \times n^{N}}: F(\xi)=0, \Phi(\xi) \leq 0\right\} .
$$

The general case follows as in [6]. 
Step 1: We first prove that

$$
\text { Rco } E=\left\{\xi \in \mathbb{R}_{s}^{m \times n^{N}}: F(\xi) \leq 0, \Phi(\xi) \leq 0\right\} .
$$

Indeed call $X$ the right hand side. It is clear that $E \subset X$ and that $X$ is rank one convex; we therefore have $\operatorname{Rco} E \subset X$. We now show the reverse inclusion. Let $\xi \in X$ be fixed and assume that $F(\xi)<0$, otherwise $\xi \in E$ and the result is trivial. Since $\Phi$ is rank one affine, we have for every $\eta$, a matrix of rank one, that for every $t \in \mathbb{R}$

$$
\Phi(\xi+t \eta)=\Phi(\xi)+t\langle D \Phi(\xi) ; \eta\rangle .
$$

We therefore choose $\eta$ a matrix of rank one so that

$$
\langle D \Phi(\xi) ; \eta\rangle=0
$$

(in the preceding theorem $\Phi(\xi)=-\operatorname{det} \xi+\delta$ and $D \Phi(\xi)=-a d j_{n-1} \xi$ ) and this leads to the desired identity

$$
\Phi(\xi+t \eta)=\Phi(\xi), \forall t \in \mathbb{R} .
$$

By compactness of $E$ we deduce that we can find $t_{1}<0<t_{2}$ so that

$$
\left\{\begin{array}{l}
F(\xi+t \eta)<0, \forall t \in\left(t_{1}, t_{2}\right) \\
F\left(\xi+t_{i} \eta\right)=0, i=1,2
\end{array}\right.
$$

We can therefore rewrite

$$
\xi=\frac{t_{2}}{t_{2}-t_{1}}\left(\xi+t_{1} \eta\right)+\frac{-t_{1}}{t_{2}-t_{1}}\left(\xi+t_{2} \eta\right)
$$

which leads to $\xi \in$ Rco $E$. Note for further reference that we easily obtain

$$
\text { int Rco } E \subset\left\{\xi \in \mathbb{R}_{s}^{m \times n^{N}}: F(\xi) \leq 0, \Phi(\xi)<0\right\} .
$$

Step 2: We wish now to show that Rco $E$ has the relaxation property with respect to $E$, i.e. that for every bounded open set $\Omega \subset \mathbb{R}^{n}$, for every $u_{\xi}$, an affine function with $D^{N} u_{\xi}(x)=\xi$, satisfying

$$
D^{N} u_{\xi}(x) \in \operatorname{int} \operatorname{Rco} E,
$$

there exists a sequence $u_{\nu} \in C_{\text {piec }}^{N}\left(\bar{\Omega} ; \mathbb{R}^{m}\right)$ such that

$$
\begin{aligned}
& u_{\nu} \in u_{\xi}+W_{0}^{N, \infty}\left(\Omega ; \mathbb{R}^{m}\right) \\
& u_{\nu} \stackrel{*}{*} u_{\xi} \text { in } W^{N, \infty} \\
& D^{N} u_{\nu}(x) \in \operatorname{int} \operatorname{Rco} E, \text { a.e in } \Omega \\
& \int_{\Omega} F\left(D^{N} u_{\nu}(x)\right) d x \rightarrow 0 \text { as } \nu \rightarrow \infty .
\end{aligned}
$$

If $F(\xi)=0$, we choose $u_{\nu}=u_{\xi}$. So from now on we can assume that $F(\xi)<0$ and $\Phi(\xi)<0$. Using then the compactness assumption we 
can find as in Step 1, $\eta$ a matrix of rank one, $t_{1}<0<t_{2}$ such that (we let $\left.\xi_{t}=\xi+t \eta\right)$

$$
\begin{aligned}
\Phi(\xi+t \eta) & =\Phi(\xi)<0 \\
F(\xi+t \eta) & =F(\xi+t \eta)<0, \forall t \in\left(t_{1}, t_{2}\right) \\
F\left(\xi+t_{1} \eta\right) & =F\left(\xi+t_{2} \eta\right)=0 .
\end{aligned}
$$

The approximation lemma (c.f. Lemma 6.8 of Dacorogna-Marcellini [6]) with $A=\xi_{t_{1}+\varepsilon}$ and $B=\xi_{t_{2}-\varepsilon}$ for $\varepsilon$ small enough and $\xi=$ $\frac{t_{2}-\varepsilon}{t_{2}-t_{1}-2 \varepsilon} A+\frac{-\left(t_{1}+\varepsilon\right)}{t_{2}-t_{1}-2 \varepsilon} B$ leads immediately to the result. Note that in this Lemma since $\operatorname{rank}[A-B]=1$

$$
\Phi(A)=\Phi(B)=\Phi(\xi)<0
$$

and the constructed function satisfies

$$
\operatorname{dist}\left(D^{N} u_{\nu}(x), \operatorname{co}\{A, B\}\right) \leq \varepsilon \quad \text { a.e. in } \Omega
$$

we deduce that by choosing $\varepsilon$ sufficiently small we have $\Phi\left(D u_{\nu}\right)<0$ and $F\left(D u_{\nu}\right)<0$ which implies that

$$
D^{N} u_{\nu}(x) \in \operatorname{int} \operatorname{Rco} E
$$

as wished. We may then apply Theorem 2.2 to get the result.

The more difficult question is to know when the relaxation property holds if the set $E$ is given by more than one equation. One such case is the following theorem that uses the notion of approximation property (c.f. Theorem 6.14 in [6]).

Definition 2.7 (Approximation property). Let $E \subset K(E) \subset \mathbb{R}^{n} \times$ $\mathbb{R}_{s}^{m \times M} \times \mathbb{R}_{s}^{m \times n^{N}}$. The sets $E$ and $K(E)$ are said to have the approximation property if there exists a family of closed sets $E_{\delta}$ and $K\left(E_{\delta}\right)$, $\delta>0$, such that

(1) $E_{\delta} \subset K\left(E_{\delta}\right) \subset \operatorname{int} K(E)$ for every $\delta>0$;

(2) for every $\varepsilon>0$ there exists $\delta_{0}=\delta_{0}(\varepsilon)>0$ such that $\operatorname{dist}(\eta ; E) \leq$ $\varepsilon$ for every $\eta \in E_{\delta}$ and $\delta \in\left[0, \delta_{0}\right]$;

(3) if $\eta \in \operatorname{int} K(E)$ then $\eta \in K\left(E_{\delta}\right)$ for every $\delta>0$ sufficiently small.

Theorem 2.8. Let $E \subset \mathbb{R}^{n} \times \mathbb{R}_{s}^{m \times M} \times \mathbb{R}_{s}^{m \times n^{N}}$ be closed and bounded uniformly with respect to $x \in \mathbb{R}^{n}$ and whenever $s$ vary on a bounded set of $\mathbb{R}_{s}^{m \times M}$ and $\mathrm{Rco} E$ has the approximation property with $K\left(E_{\delta}\right)=$ Rco $E_{\delta}$, then it has the relaxation property with respect to $E$.

As a corollary we obtain (c.f. Corollary 6.18 in [6] or [7]). 
Corollary 2.9. Let $\Omega \subset \mathbb{R}^{n}$ be open. Let $F_{i}: \mathbb{R}_{s}^{m \times n^{N}} \rightarrow \mathbb{R}, i=$ $1,2, \ldots, I$, be quasiconvex and let

$$
E=\left\{\begin{array}{c}
\xi \in \mathbb{R}_{s}^{m \times n^{N}}: F_{i}(\xi)=0, \quad i=1,2, \ldots, I_{1} \\
F_{i}(\xi) \leq 0, i=I_{1}+1, \ldots, I
\end{array}\right\} .
$$

Assume that Rco $E$ is compact and Rco $E=\operatorname{co} E$. Let $\varphi \in C_{p i e c}^{N}\left(\bar{\Omega} ; \mathbb{R}^{m}\right)$ verify

$$
\begin{gathered}
D^{N} \varphi(x) \in E \cup \operatorname{int} \operatorname{Rco} E, \text { a.e. } x \in \Omega, \\
\text { or } \varphi \in W^{N, \infty}\left(\Omega ; \mathbb{R}^{m}\right) \text { satisfy } \\
D^{N} \varphi(x) \text { compactly contained in int Rco } E \text {, a.e. } x \in \Omega .
\end{gathered}
$$

Then there exists (a dense set of) $u \in \varphi+W_{0}^{N, \infty}\left(\Omega ; \mathbb{R}^{m}\right)$ such that

$$
\left\{\begin{array}{l}
F_{i}\left(D^{N} u(x)\right)=0, \quad \text { a.e. } x \in \Omega, \quad i=1, \ldots, I_{1} \\
F_{i}\left(D^{N} u(x)\right) \leq 0, \quad \text { a.e. } x \in \Omega, \quad i=I_{1}+1, \ldots, I .
\end{array}\right.
$$

\section{The incompressible CASE}

Comparable results to those of the present Section are obtained by Müller-Sverak [14] using the ideas of Gromov on convex integration; here we show how the method of Dacorogna-Marcellini in [6] can also be applied.

In the present Section and in Section 9 and 10 we will consider subsets $E$ or $K$ of the manifold $\operatorname{det} \xi=1$, so when we will write int $K$ we will mean the interior relative to the manifold.

We now adapt the definitions of the relaxation and the approximation properties to the present context. Here we give the first one under a slightly more restrictive form in order to avoid some technicalities. We first let for $\theta>0$ and $\Omega \subset \mathbb{R}^{n}$ an open set, $W_{\theta}$ be the set of functions $u \in C_{\text {piec }}^{1}\left(\bar{\Omega} ; \mathbb{R}^{n}\right)$ such that there exists an open set $\Omega_{\theta} \subset \Omega$ so that meas $\left(\Omega-\Omega_{\theta}\right)<\theta$ and $u$ is piecewise affine in $\Omega_{\theta}$. We could consider a more general set but the proof is then more involved.

Definition 3.1 (Relaxation property). Let $E, K \subset \mathbb{R}^{n} \times \mathbb{R}^{n} \times \mathbb{R}^{n \times n}$. We say that $K$ has the relaxation property with respect to $E$ if for every bounded open set $\Omega \subset \mathbb{R}^{n}$, for every $u_{\xi}$, an affine function with $D u_{\xi}(x)=\xi$, satisfying

$$
\left(x, u_{\xi}(x), D u_{\xi}(x)\right) \in \operatorname{int} K,
$$


there exists a sequence $u_{\nu} \in W_{1 / \nu}$ such that

$$
\begin{aligned}
& u_{\nu} \in u_{\xi}+W_{0}^{1, \infty}\left(\Omega ; \mathbb{R}^{n}\right) \\
& u_{\nu} \stackrel{*}{*} u_{\xi} \text { in } W^{1, \infty} \\
& \left(x, u_{\nu}(x), D u_{\nu}(x)\right) \in E \cup \operatorname{int} K, \text { a.e in } \Omega \\
& \int_{\Omega} \operatorname{dist}\left(\left(x, u_{\nu}(x), D u_{\nu}(x)\right) ; E\right) d x \rightarrow 0 \text { as } \nu \rightarrow \infty .
\end{aligned}
$$

The following theorem is the main abstract existence theorem.

Theorem 3.2. Let $\Omega \subset \mathbb{R}^{n}$ be open. Let $F_{i}: \Omega \times \mathbb{R}^{n} \times \mathbb{R}^{n \times n} \rightarrow \mathbb{R}, F_{i}=$ $F_{i}(x, s, \xi), i=1,2, \ldots, I$, be continuous with respect to all variables and quasiconvex with respect to the variable $\xi$. Let $E \subset \mathbb{R}^{n} \times \mathbb{R}^{n} \times \mathbb{R}^{n \times n}$ be such that

$$
E=\left\{\begin{array}{c}
(x, s, \xi) \in \mathbb{R}^{n} \times \mathbb{R}^{n} \times \mathbb{R}^{n \times n}: F_{i}(x, s, \xi)=0, i=1,2, \ldots, I_{1} \\
F_{i}(x, s, \xi) \leq 0, i=I_{1}+1, \ldots, I, \operatorname{det} \xi=1
\end{array}\right\} .
$$

Assume that Rco $E$ has the relaxation property with respect to $E$ and that it is bounded uniformly for $x \in \Omega$ and whenever $s$ vary on a bounded set of $\mathbb{R}^{n \times n}$. Let $\varphi$ be an affine function such that

$$
(x, \varphi(x), D \varphi(x)) \in E \cup \operatorname{int} \operatorname{Rco} E \text {, in } \Omega \text {; }
$$

then there exists (a dense set of) $u \in \varphi+W_{0}^{1, \infty}\left(\Omega ; \mathbb{R}^{n}\right)$ such that

$$
\left\{\begin{array}{l}
F_{i}(x, u(x), D u(x))=0, i=1,2, \ldots, I_{1}, \text { a.e. } x \in \Omega \\
F_{i}(x, u(x), D u(x)) \leq 0, i=I_{1}+1, \ldots, I, \text { a.e. } x \in \Omega \\
\operatorname{det} D u(x)=1, \text { a.e. } x \in \Omega .
\end{array}\right.
$$

Proof. We will make the proof when there is no dependence on lower order terms, otherwise use the standard procedure in [6].

Step 1: We first observe that $\Omega \subset \mathbb{R}^{n}$ can be assumed bounded, without loss of generality. We then let $V$ be the set of functions $u$ so that there exists $u_{\nu} \in W_{1 / \nu}, u_{\nu}=\varphi$ on $\partial \Omega$ and $D u_{\nu} \in E \cup \operatorname{int} \operatorname{Rco} E$ a.e. such that $u_{\nu} \rightarrow u$ in $L^{\infty}(\Omega)$ as $\nu \rightarrow \infty$.

Note that $\varphi \in V$ and $V$ is a complete metric space when endowed with the $C^{0}$ norm. Note that by weak lower semicontinuity we have

$$
V \subset\left\{\begin{array}{c}
u \in \varphi+W_{0}^{1, \infty}\left(\Omega ; \mathbb{R}^{n}\right): \\
F_{i}(D u(x)) \leq 0, i=1,2, \ldots, I, \text { a.e. } x \in \Omega \\
\operatorname{det} D u(x)=1, \text { a.e. } x \in \Omega
\end{array}\right\}
$$

Step 2: Let for $u \in V$

$$
L(u)=\sum_{i=1}^{I_{1}} \int_{\Omega} F_{i}(D u(x)) d x .
$$


Observe that by quasiconvexity of $F_{i}$ we have for every $u \in V$

$$
\liminf _{u_{s} * u, u_{s} \in V} L\left(u_{s}\right) \geq L(u) .
$$

We next immediately see that for every $u \in V$ (recall that in $V$ we have $\operatorname{det} D u=1$ and $\left.F_{i}(D u) \leq 0, i=I_{1}+1, \ldots, I\right)$

$$
L(u)=0 \Leftrightarrow D u(x) \in E \text {, a.e. in } \Omega .
$$

We then let

$$
V^{k}=\left\{u \in V: L(u)>-\frac{1}{k}\right\} .
$$

We have that $V^{k}$ is open (c.f. (3)). Furthermore it is dense in $V$. This will be proved in Step 3. If this property has been established we deduce from Baire category theorem that $\cap V^{k}$ is dense in $V$. Thus the result by (4).

Step 3: So it remains to prove that for any $u \in V$ and any $\varepsilon>0$ sufficiently small we can find $u_{\varepsilon} \in V^{k}$ so that

$$
\left\|u_{\varepsilon}-u\right\|_{\infty} \leq \varepsilon .
$$

We will prove this property under the further assumption that, for some $\theta>0$, small, $u \in W_{\theta}$ and

$$
D u(x) \in E \cup \operatorname{int} \operatorname{Rco} E \text {, a.e in } \Omega \text {. }
$$

The general case will follow by definition of $V$. By working on each piece where $u$ is affine and by setting $u_{\varepsilon}=u$ on $\Omega-\Omega_{\theta}$ we can assume that $u$ is affine in $\Omega$. The result now follows at once from the relaxation property.

As usual if the set $E$ is defined by only one equation the relaxation property is easier to establish and we therefore have as a first consequence of the theorem.

Theorem 3.3. Let $\Omega \subset \mathbb{R}^{n}$ be open. Let $F: \Omega \times \mathbb{R}^{n} \times \mathbb{R}^{n \times n} \rightarrow \mathbb{R}$ be continuous and quasiconvex and coercive, with respect to the last variable $\xi$, in any direction, uniformly with respect to $x \in \Omega$ and $s$ in a bounded set of $\mathbb{R}^{n}$. If $\varphi$ is affine and is such that

$$
\left\{\begin{array}{c}
F(x, \varphi(x), D \varphi(x)) \leq 0, \text { a.e. } x \in \Omega, \\
\operatorname{det} D \varphi(x)=1, \text { a.e. } x \in \Omega,
\end{array}\right.
$$

then there exists (a dense set of) $u \in \varphi+W_{0}^{1, \infty}\left(\Omega ; \mathbb{R}^{n}\right)$ such that

$$
\left\{\begin{array}{l}
F(x, u(x), D u(x))=0, \text { a.e. } x \in \Omega \\
\operatorname{det} D u(x)=1, \text { a.e. } x \in \Omega .
\end{array}\right.
$$


Proof. We first let

$$
\begin{aligned}
& \Omega_{0}=\{x \in \Omega: F(x, \varphi(x), D \varphi(x))=0\} \\
& \Omega_{1}=\Omega-\Omega_{0}=\{x \in \Omega: F(x, \varphi(x), D \varphi(x))<0\}
\end{aligned}
$$

and observe that, by continuity, $\Omega_{0}$ is closed and hence $\Omega_{1}$ is open; we therefore need only to work on this last set, since in $\Omega_{0}$ we can choose $u=\varphi$.

We may now apply the abstract theorem with

$$
E=\left\{(x, s, \xi) \in \mathbb{R}^{n} \times \mathbb{R}^{n} \times \mathbb{R}^{n \times n}: F(x, s, \xi)=0, \operatorname{det} \xi=1\right\} .
$$

$K=\operatorname{Rco} E=\left\{(x, s, \xi) \in \mathbb{R}^{n} \times \mathbb{R}^{n} \times \mathbb{R}^{n \times n}: F(x, s, \xi) \leq 0, \operatorname{det} \xi=1\right\}$. The proposition below ensures that all the hypotheses of the abstract theorem are satisfied and therefore the theorem is proved.

Proposition 3.4. Let $F: \mathbb{R}^{n} \times \mathbb{R}^{n} \times \mathbb{R}^{n \times n} \rightarrow \mathbb{R}$ be continuous and rank one convex and coercive, with respect to the last variable $\xi$, in any direction, uniformly with respect to $x \in \Omega$ and $s$ in a bounded set of $\mathbb{R}^{n}$. Let

$$
E=\left\{(x, s, \xi) \in \mathbb{R}^{n} \times \mathbb{R}^{n} \times \mathbb{R}^{n \times n}: F(x, s, \xi)=0, \operatorname{det} \xi=1\right\} .
$$

Then

$$
\operatorname{Rco} E=\left\{(x, s, \xi) \in \mathbb{R}^{n} \times \mathbb{R}^{n} \times \mathbb{R}^{n \times n}: F(x, s, \xi) \leq 0, \operatorname{det} \xi=1\right\} \text {. }
$$

Furthermore Rco $E$ has the relaxation property with respect to $E$.

Proof. We will do the proof when there is no dependence on lower order terms, i.e.

$$
E=\left\{\xi \in \mathbb{R}^{n \times n}: F(\xi)=0, \operatorname{det} \xi=1\right\} .
$$

Step 1 : We now prove that

$$
\text { Rco } E=\left\{\xi \in \mathbb{R}^{n \times n}: F(\xi) \leq 0, \operatorname{det} \xi=1\right\} \text {. }
$$

Indeed call $X$ the right hand side. It is clear that $E \subset X$ and that $X$ is rank one convex; we therefore have $\operatorname{Rco} E \subset X$. We now show the reverse inclusion. Let $\xi \in X$ be fixed and assume that $F(\xi)<0$, otherwise $\xi \in E$ and the result is trivial. We can then find $\eta \in \mathbb{R}^{n \times n}$ a matrix of rank one so that

$$
\operatorname{det}(\xi+t \eta)=\operatorname{det} \xi=1
$$

for every $t \in \mathbb{R}$. This is easy and follows from the following observation ( $\eta$ being a matrix of rank one)

$$
\operatorname{det}(\xi+t \eta)=\operatorname{det} \xi+t\left\langle a d j_{n-1} \xi ; \eta\right\rangle ;
$$

so choose $\eta$ so that

$$
\left\langle a d j_{n-1} \xi ; \eta\right\rangle=0
$$


By compactness of $E$ we deduce that we can find $t_{1}<0<t_{2}$ so that

$$
\left\{\begin{array}{l}
F(\xi+t \eta)<0, \forall t \in\left(t_{1}, t_{2}\right) \\
F\left(\xi+t_{i} \eta\right)=0, i=1,2
\end{array}\right.
$$

We can therefore rewrite

$$
\xi=\frac{t_{2}}{t_{2}-t_{1}}\left(\xi+t_{1} \eta\right)+\frac{-t_{1}}{t_{2}-t_{1}}\left(\xi+t_{2} \eta\right)
$$

which leads to $\xi \in$ Rco $E$.

Step 2: We wish now to show that for every bounded open set $\Omega \subset \mathbb{R}^{n}$, for every $u_{\xi}$, an affine function with $D u_{\xi}(x)=\xi$, satisfying

$$
D u_{\xi}(x) \in \operatorname{int} \operatorname{Rco} E,
$$

there exists a sequence $u_{\nu} \in W_{1 / \nu}$, such that

$$
\begin{aligned}
& u_{\nu} \in u_{\xi}+W_{0}^{1, \infty}\left(\Omega ; \mathbb{R}^{n}\right) \\
& u_{\nu} \stackrel{*}{\rightarrow} u_{\xi} \text { in } W^{1, \infty} \\
& D u_{\nu}(x) \in \operatorname{int} \operatorname{Rco} E, \text { a.e in } \Omega \\
& \int_{\Omega} F\left(D u_{\nu}(x)\right) d x \rightarrow 0 \text { as } \nu \rightarrow \infty .
\end{aligned}
$$

If $F(\xi)=0$, we choose $u_{\nu}=u_{\xi}$. So from now on we can assume that $F(\xi)<0$ (and $\operatorname{det} \xi=1$ ). Using then the coercivity assumption we can find as in Step 1, $\eta$ a matrix of rank one, $t_{1}<0<t_{2}$ such that (we let $\xi_{t}=\xi+t \eta$ )

$$
\begin{aligned}
\operatorname{det}(\xi+t \eta) & =\operatorname{det} \xi=1 \\
F(\xi+t \eta) & =F(\xi+t \eta)<0, \forall t \in\left(t_{1}, t_{2}\right) \\
F\left(\xi+t_{1} \eta\right) & =F\left(\xi+t_{2} \eta\right)=0 .
\end{aligned}
$$

So let $\nu$ be large enough. We can then find $\delta=\delta(\nu)>0$ so that

$$
F\left(\xi_{t}\right) \leq-\delta, \quad \forall t \in\left[t_{1}+1 / \nu, t_{2}-1 / \nu\right] .
$$

Call $A=\xi_{t_{1}+1 / \nu}, B=\xi_{t_{2}-1 / \nu}, \varphi(x)=u_{\xi}(x)$ and observe that

$$
\operatorname{det} A=\operatorname{det} B=1, \quad \operatorname{rank}\{A-B\}=1
$$

and

$$
D \varphi=\xi=\frac{t_{2}-1 / \nu}{t_{2}-t_{1}-2 / \nu} A+\frac{-\left(t_{1}+1 / \nu\right)}{t_{2}-t_{1}-2 / \nu} B .
$$

By continuity of $F$ we can find $\delta^{\prime}=\delta^{\prime}(\delta=\delta(\nu))>0$ so that

$$
\operatorname{dist}(\eta, \operatorname{co}\{A, B\}) \leq \delta^{\prime} \Rightarrow F(\eta) \leq-\delta / 2 .
$$

Therefore apply approximation Lemma 4.1 with $\varepsilon<\min \left\{1 / \nu, \delta^{\prime}\right\}$ and call $u_{\nu}$ the function that is found in the Lemma, to get the result. 
The next question we discuss is to know when the relaxation property holds if the set $E$ is defined by more than one equation. The question is more involved and we need, as in Section 2 or as in [6], the so called approximation property.

Definition 3.5 (Approximation property). Let $E \subset K(E) \subset \mathbb{R}^{n} \times$ $\mathbb{R}^{n} \times \mathbb{R}^{n \times n}$. The sets $E$ and $K(E)$ are said to have the approximation property if there exists a family of closed sets $E_{\delta}$ and $K\left(E_{\delta}\right), \delta>0$, such that

(1) $E_{\delta} \subset K\left(E_{\delta}\right) \subset \operatorname{int} K(E)$ for every $\delta>0$;

(2) for every $\varepsilon>0$ there exists $\delta_{0}=\delta_{0}(\varepsilon)>0$ such that $\operatorname{dist}(\eta ; E) \leq$ $\varepsilon$ for every $\eta \in E_{\delta}$ and $\delta \in\left[0, \delta_{0}\right]$;

(3) if $\eta \in \operatorname{int} K(E)$ then $\eta \in K\left(E_{\delta}\right)$ for every $\delta>0$ sufficiently small.

Theorem 3.6. Let $E \subset \mathbb{R}^{n} \times \mathbb{R}^{n} \times \mathbb{R}^{n \times n}$ be closed and bounded uniformly with respect to $x \in \mathbb{R}^{n}$ and whenever $s$ vary on a bounded set of $\mathbb{R}^{n}$ and Rco $E$ has the approximation property with $K\left(E_{\delta}\right)=\operatorname{Rco} E_{\delta}$, then it has the relaxation property with respect to $E$.

Proof. We will make the proof when there is no dependence on lower order terms. We therefore are given $\Omega \subset \mathbb{R}^{n}$, a bounded open set and $u$, an affine function with $D u(x)=\xi$, with $\xi \in \operatorname{int}$ Rco $E$ and we wish to show that there exists a sequence $u_{\varepsilon} \in W_{\varepsilon}$ such that

$$
\begin{aligned}
& u_{\varepsilon} \in u+W_{0}^{1, \infty}\left(\Omega ; \mathbb{R}^{n}\right) \\
& u_{\varepsilon} * \text { in } W^{1, \infty} \\
& D u_{\varepsilon}(x) \in E \cup \text { int Rco } E, \text { a.e in } \Omega \\
& \int_{\Omega} \operatorname{dist}\left(D u_{\varepsilon}(x) ; E\right) d x \rightarrow 0 \text { as } \varepsilon \rightarrow 0 .
\end{aligned}
$$

By the approximation property we have, for some $\delta$, that $\xi \in \operatorname{Rco} E_{\delta}$ and hence that $\xi \in R_{J}$ co $E_{\delta}$ for a certain $J$. We then proceed by induction on $J$.

Step 3.1: We start with $J=1$. We can therefore write

$$
D u=\xi=t A+(1-t) B, \operatorname{rank}\{A-B\}=1,
$$

with

$$
A, B \in E_{\delta}
$$


We then use the approximation Lemma 4.1 to find (setting $\Omega_{\varepsilon}=\Omega_{A} \cup$ $\left.\Omega_{B}\right) u_{\varepsilon} \in W_{\varepsilon}$

$$
\left\{\begin{array}{l}
u_{\varepsilon} \equiv u \text { near } \partial \Omega \\
\left\|u_{\varepsilon}-u\right\|_{\infty} \leq \varepsilon \\
D u_{\varepsilon}(x)=\left\{\begin{array}{l}
A \text { in } \Omega_{A} \\
B \text { in } \Omega_{B}
\end{array}\right. \\
\operatorname{det} D u_{\varepsilon}(x)=\operatorname{det} A=\operatorname{det} B, \quad \text { in } \Omega \\
\operatorname{dist}\left(D u_{\varepsilon}(x) ; \operatorname{Rco} E_{\delta}\right) \leq \varepsilon, \quad \text { in } \Omega
\end{array}\right.
$$

where we have used the fact that

$$
\operatorname{co}\{A, B\} \subset \operatorname{Rco} E_{\delta} .
$$

The claim (6) follows by choosing $\varepsilon$ and $\delta$ smaller if necessary.

Step 3.2: We now let for $J>1$

$$
\xi \in R_{J} \operatorname{co} E_{\delta}
$$

Therefore there exist $A, B \in \mathbb{R}^{n \times n}$ such that

$$
\left\{\begin{array}{l}
\xi=t A+(1-t) B, \quad \operatorname{rank}\{A-B\}=1 \\
A, B \in R_{J-1} \operatorname{co} E_{\delta} .
\end{array}\right.
$$

We then apply the approximation Lemma 4.1 and find that there exist a vector valued function $v \in W_{\varepsilon / 2}$ and $\Omega_{A}, \Omega_{B}$ disjoint open sets such that

$$
\left\{\begin{array}{l}
\text { meas }\left(\Omega-\left(\Omega_{A} \cup \Omega_{B}\right)\right) \leq \varepsilon / 2 . \text { meas } \Omega \\
v \equiv u \text { near } \partial \Omega \\
\|v-u\|_{\infty} \leq \varepsilon / 2 \\
D v(x)=\left\{\begin{array}{l}
A \text { in } \Omega_{A} \\
B \text { in } \Omega_{B}
\end{array}\right. \\
\operatorname{dist}\left(D v(x) ; \operatorname{Rco} E_{\delta}\right) \leq \varepsilon, \text { in } \Omega .
\end{array}\right.
$$

We now use the hypothesis of induction on $\Omega_{A}, \Omega_{B}$ and $A, B$. We then can find $\widetilde{\Omega}_{A}, \widetilde{\Omega}_{B}, v_{A} \in W_{\varepsilon / 4}$ in $\Omega_{A}, v_{B} \in W_{\varepsilon / 4}$ in $\Omega_{B}$ satisfying

$$
\left\{\begin{array}{l}
\operatorname{meas}\left(\Omega_{A}-\widetilde{\Omega}_{A}\right), \operatorname{meas}\left(\Omega_{B}-\widetilde{\Omega}_{B}\right) \leq \varepsilon / 4 . \text { meas } \Omega \\
v_{A} \equiv v \text { near } \partial \Omega_{A}, v_{B} \equiv v \text { near } \partial \Omega_{B} \\
\left\|v_{A}-v\right\|_{\infty} \leq \varepsilon / 2 \text { in } \bar{\Omega}_{A},\left\|v_{B}-v\right\|_{\infty} \leq \varepsilon / 2 \text { in } \bar{\Omega}_{B} \\
\operatorname{dist}\left(D v_{A}(x) ; E_{\delta}\right) \leq \varepsilon, \text { a.e. in } \widetilde{\Omega}_{A}, \\
\operatorname{dist}\left(D v_{B}(x) ; E_{\delta}\right) \leq \varepsilon, \text { a.e. in } \widetilde{\Omega}_{B} \\
\operatorname{dist}\left(D v_{A}(x) ; \operatorname{Rco} E_{\delta}\right) \leq \varepsilon, \text { a.e. in } \Omega_{A} \\
\operatorname{dist}\left(D v_{B}(x) ; \operatorname{Rco} E_{\delta}\right) \leq \varepsilon, \text { a.e. in } \Omega_{B} .
\end{array}\right.
$$


Letting $\widetilde{\Omega}=\widetilde{\Omega}_{A} \cup \widetilde{\Omega}_{B}$ and

$$
u_{\varepsilon}(x)= \begin{cases}v(x) & \text { in } \Omega-\left(\Omega_{A} \cup \Omega_{B}\right) \\ v_{A}(x) & \text { in } \Omega_{A} \\ v_{B}(x) & \text { in } \Omega_{B}\end{cases}
$$

we have indeed obtained (6) by choosing $\varepsilon$ and $\delta$ smaller if necessary, and thus the result.

\section{The approximation lemma}

The following result is due to Müller-Sverak [14] and is an extension of a classical lemma (c.f. for example Lemma 6.8 in [6]) to handle constraint on the determinants. For the convenience of the reader we will give the proof of Müller-Sverak with however a slight variation.

Lemma 4.1. Let $\Omega \subset \mathbb{R}^{n}$ be an open set with finite measure. Let $t \in$ $[0,1]$ and $A, B \in \mathbb{R}^{n \times n}$ with $\operatorname{rank}\{A-B\}=1$ and $\operatorname{det} A=\operatorname{det} B>0$. Let $\varphi$ be such that

$$
D \varphi(x)=t A+(1-t) B, \quad \forall x \in \bar{\Omega} .
$$

Then, for every $\varepsilon>0$, there exist $u \in C^{\infty}\left(\bar{\Omega} ; \mathbb{R}^{n}\right)$ and disjoint open sets $\Omega_{A}, \Omega_{B} \subset \Omega$, so that

$$
\left\{\begin{array}{l}
\mid \text { meas } \Omega_{A}-t \text { meas } \Omega|,| \text { meas } \Omega_{B}-(1-t) \text { meas } \Omega \mid \leq \varepsilon \\
u \equiv \varphi \text { near } \partial \Omega \\
\|u-\varphi\|_{\infty} \leq \varepsilon \\
D u(x)= \begin{cases}A & \text { in } \Omega_{A} \\
B \quad \text { in } \Omega_{B}\end{cases} \\
\operatorname{det} D u(x)=\operatorname{det} A=\operatorname{det} B, \quad \text { in } \Omega \\
\operatorname{dist}(D u(x), \operatorname{co}\{A, B\}) \leq \varepsilon, \quad \text { in } \Omega .
\end{array}\right.
$$

Remark 4.2. By co $\{A, B\}=[A, B]$ we mean the closed segment joining $A$ to $B$.

Proof. We divide the proof into two steps.

Step 1: We first assume that

$$
D \varphi=t A+(1-t) B=I
$$

and hence $\operatorname{det} A=\operatorname{det} B=1$. We also assume (these assumptions will be removed in Step 2) that the matrix has the form

$$
A-B=\alpha \otimes e_{1}
$$


where $e_{1}=(1,0, \ldots, 0), \alpha=\left(0, \alpha_{2}, \ldots, \alpha_{n}\right) \in \mathbb{R}^{n}$, i.e.

$$
A-B=\left(\begin{array}{cccc}
0 & 0 & \ldots & 0 \\
\alpha_{2} & 0 & \ldots & 0 \\
\ldots & \ldots & \ldots & \ldots \\
\alpha_{n} & 0 & \ldots & 0
\end{array}\right) \in \mathbb{R}^{n \times n} .
$$

We can express $\Omega$ as union of cubes with faces parallel to the coordinate axes and a set of small measure. Then, by posing $u \equiv \varphi$ on the set of small measure, and by homotheties and translations, we can reduce ourselves to work with $\Omega$ equal to the unit cube.

Let $\Omega_{\varepsilon}$ be a set compactly contained in $\Omega$ and let $\eta \in C_{0}^{\infty}(\Omega)$ and $L>0$ be such that

$$
\left\{\begin{array}{l}
\text { meas }\left(\Omega-\Omega_{\varepsilon}\right) \leq \varepsilon / 2 \\
0 \leq \eta(x) \leq 1, \quad \forall x \in \Omega \\
\eta(x)=1, \quad \forall x \in \Omega_{\varepsilon} \\
|D \eta(x)| \leq L / \varepsilon, \quad \forall x \in \Omega-\Omega_{\varepsilon} \\
\left|D^{2} \eta(x)\right| \leq L / \varepsilon^{2}, \quad \forall x \in \Omega-\Omega_{\varepsilon} .
\end{array}\right.
$$

Let us define a $C^{\infty}$ function $v:[0,1] \rightarrow \mathbb{R}$ in the following way: given $\delta>0$, divide the interval $(0,1)$ into three finite unions $I_{A}, I_{B}, J$ of disjoint open subintervals such that

$$
\left\{\begin{array}{l}
\bar{I}_{A} \cup \bar{I}_{B} \cup \bar{J}=[0,1] \\
\text { meas } I_{A}=t-\delta, \text { meas } I_{B}=1-t-\delta \\
v^{\prime \prime}\left(x_{1}\right)=\left\{\begin{array}{l}
(1-t) \text { if } x_{1} \in I_{A} \\
-t \text { if } x_{1} \in I_{B}
\end{array}\right. \\
v^{\prime \prime}\left(x_{1}\right) \in[-t,(1-t)], \forall x_{1} \in(0,1) \\
\left|v\left(x_{1}\right)\right|,\left|v^{\prime}\left(x_{1}\right)\right| \leq \delta, \forall x_{1} \in(0,1) .
\end{array}\right.
$$

We then let

$$
\Omega_{A}=\left\{x \in \Omega_{\varepsilon}: x_{1} \in I_{A}\right\}, \Omega_{B}=\left\{x \in \Omega_{\varepsilon}: x_{1} \in I_{B}\right\}
$$

and observe that by choosing $\delta$ small enough we have

$$
\mid \text { meas } \Omega_{A}-t \text { meas } \Omega|,| \text { meas } \Omega_{B}-(1-t) \text { meas } \Omega \mid \leq \varepsilon \text {. }
$$

We next define $V: \mathbb{R}^{n} \rightarrow \mathbb{R}^{n}$ by

$$
\begin{aligned}
V(x)= & v^{\prime}\left(x_{1}\right) \eta(x)\left(0, \alpha_{2}, \ldots, \alpha_{n}\right) \\
& +v\left(x_{1}\right)\left(-\sum_{i=2}^{n} \alpha_{i} \frac{\partial \eta}{\partial x_{i}}, \alpha_{2} \frac{\partial \eta}{\partial x_{1}}, \alpha_{3} \frac{\partial \eta}{\partial x_{1}}, \ldots, \alpha_{n} \frac{\partial \eta}{\partial x_{1}}\right) .
\end{aligned}
$$


Note that $V \in C^{\infty}$ and has the following properties (where $\delta$ has been chosen sufficiently small)

$$
\begin{aligned}
\operatorname{div} V & \equiv 0 \text { in } \Omega \\
V & \equiv 0 \text { near } \partial \Omega \\
\left|D V-v^{\prime \prime} \eta \alpha \otimes e_{1}\right|,|V| & \leq \varepsilon^{2} \text { in } \Omega \\
D V & =\left\{\begin{array}{c}
(1-t) \alpha \otimes e_{1}, \text { in } \Omega_{A} \\
-t \alpha \otimes e_{1}, \text { in } \Omega_{B} .
\end{array}\right.
\end{aligned}
$$

We can now define $u$ as the flow associated to the vector field $V$ (this is the usual procedure to construct a volume preserving map), i.e.

$$
\left\{\begin{array}{c}
\frac{d}{d s} u(s, x)=V(u(s, x)), s \in[0,1] \\
u(0, x)=x .
\end{array}\right.
$$

The map $u(x)=u(1, x)$ has all the claimed properties, as will now be shown.

1) Indeed since $V \equiv 0$ near $\partial \Omega$, we have by uniqueness of the solution of the differential system that

$$
u(s, x) \equiv x, \forall s \in[0,1]
$$

and hence the boundary condition for $u$ is satisfied (recall that by hypothesis we are considering the case $\varphi(x)=x)$.

2) Since we have $|V| \leq \varepsilon^{2}$ we deduce that

$$
|u(s, x)-x|=\left|\int_{0}^{s} V(u(\sigma, x)) d \sigma\right| \leq \varepsilon^{2} s, \forall s \in[0,1], \forall x \in \Omega .
$$

3) If $x \in \Omega_{A} \cup \Omega_{B}$ then by uniqueness of solutions we find

$$
u(s, x)=x+s v^{\prime}\left(x_{1}\right) \alpha, \forall s \in[0,1]
$$

and hence

$$
D u(x)=D_{x} u(1, x)=I+v^{\prime \prime}\left(x_{1}\right) \alpha \otimes e_{1}= \begin{cases}A & \text { in } \Omega_{A} \\ B & \text { in } \Omega_{B} .\end{cases}
$$

4) Since $\operatorname{div} V \equiv 0$ in $\Omega$ we have automatically (c.f. for example [2] page 28)

$$
\operatorname{det} D_{x} u(s, x) \equiv 1, \forall s \in[0,1], \forall x \in \Omega .
$$

5) Finally it remains to show that

$$
\operatorname{dist}(D u(x), \operatorname{co}\{A, B\}) \leq \varepsilon \quad \text { a.e. in } \Omega \text {. }
$$

We first set

$$
L(x)=v^{\prime \prime}\left(x_{1}\right) \eta(x) \alpha \otimes e_{1} .
$$


Returning to (8) we get

$$
\left\{\begin{array}{c}
\frac{d}{d s} D_{x} u(s, x)=D V(u(s, x)) D_{x} u(s, x), s \in[0,1] \\
D_{x} u(0, x)=I
\end{array}\right.
$$

and we compare the solution of this system with the one of

$$
\left\{\begin{array}{c}
\frac{d}{d s} F(s, x)=L(x) F(s, x), s \in[0,1] \\
F(0, x)=I .
\end{array}\right.
$$

Using the properties of $V$ and (9) we get that

$$
\left|F(s, x)-D_{x} u(s, x)\right| \leq \varepsilon, \forall s \in[0,1], \forall x \in \Omega .
$$

The conclusion then follows from the observation that

$$
F(s, x)=e^{s L(x)}=I+s v^{\prime \prime}\left(x_{1}\right) \eta(x) \alpha \otimes e_{1}, s \in[0,1]
$$

and the facts that $\eta \in[0,1], v^{\prime \prime} \in[-t,(1-t)]$.

Step 2: We now consider the general case. Since $A-B$ is a matrix of rank one we can find $a, b \in \mathbb{R}^{n}$ (replacing $a$ by $|b| a$ we can assume that $|b|=1$ ) such that

$$
C^{-1} A-C^{-1} B=a \otimes b .
$$

where $C=D \varphi=t A+(1-t) B$. We can then find $R=\left(r_{i j}\right) \in$ $S O(n) \subset \mathbb{R}^{n \times n}$ (i.e. a rotation) so that $b=e_{1} R$ and hence $e_{1}=b R^{t}$. We then set $\widetilde{\Omega}=R \Omega$ and

$$
\widetilde{A}=R C^{-1} A R^{t} \quad \text { and } \quad \widetilde{B}=R C^{-1} B R^{t} .
$$

We observe that by construction, setting $\alpha=R a$, we have

$$
\begin{aligned}
t \widetilde{A}+(1-t) \widetilde{B} & =I \\
\widetilde{A}-\widetilde{B} & =\alpha \otimes e_{1} .
\end{aligned}
$$

Note that this implies in particular that $\alpha_{1}=0($ since $\operatorname{det} \widetilde{A}=\operatorname{det} \widetilde{B}=$ 1 ) and hence $\alpha=\left(0, \alpha_{2}, \ldots, \alpha_{n}\right)$. We may therefore apply Step 1 to $\widetilde{\Omega}$ and to $\widetilde{\varphi}(y)=R C^{-1} \varphi\left(R^{t} y\right)$ and find $\widetilde{\Omega}_{\widetilde{A}}, \widetilde{\Omega}_{\widetilde{B}}$ and $\widetilde{u} \in C^{\infty}\left(\overline{\widetilde{\Omega}} ; \mathbb{R}^{n}\right)$ with the claimed properties. By setting

$$
\left\{\begin{array}{l}
u(x)=C R^{t} \widetilde{u}(R x), \quad x \in \Omega \\
\Omega_{A}=R^{t} \widetilde{\Omega}_{\widetilde{A}}, \quad \Omega_{B}=R^{t} \widetilde{\Omega}_{\widetilde{B}}
\end{array}\right.
$$

we get the result by recalling that

$$
D u(x)=C R^{t} D \widetilde{u}(R x) R .
$$




\section{The Complex eikonal Equation}

We now discuss Example 1.1. We will first derive a theorem on the rank one convex hull and then go back to the differential equation.

First recall the notation of the Appendix. For a matrix $\xi \in \mathbb{R}^{m \times n}$

$$
\xi=\left(\begin{array}{lll}
\xi_{1}^{1} & \cdots & \xi_{n}^{1} \\
\vdots & & \vdots \\
\xi_{1}^{m} & \cdots & \xi_{n}^{m}
\end{array}\right)=\left(\begin{array}{l}
\xi^{1} \\
\vdots \\
\xi^{m}
\end{array}\right)=\left(\xi_{1}, \ldots, \xi_{n}\right)
$$

we let $0 \leq \lambda_{1}(\xi) \leq \ldots \leq \lambda_{m \wedge n}(\xi)$ be its singular values.

We then have the following

Theorem 5.1. Case 1 : Let $m \leq n, r^{1}, \ldots, r^{m}>0$ and

$$
E=\left\{\xi \in \mathbb{R}^{m \times n}:\left\langle\xi^{i} ; \xi^{j}\right\rangle=r^{i} r^{j} \delta^{i j}\right\}
$$

where $\delta^{i j}$ is the Kronecker symbol. Let

$$
A=\operatorname{diag}\left(\frac{1}{r^{1}}, \ldots, \frac{1}{r^{m}}\right) \in \mathbb{R}^{m \times m}
$$

then

$$
\begin{aligned}
E & =\left\{\xi \in \mathbb{R}^{m \times n}: \lambda_{i}(A \xi)=1, i=1, \ldots, m\right\} \\
\operatorname{Rco} E & =\operatorname{co} E=\left\{\xi \in \mathbb{R}^{m \times n}: \lambda_{m}(A \xi) \leq 1\right\} .
\end{aligned}
$$

Case 2: Let $m \geq n, r_{1}, \ldots, r_{n}>0$ and

$$
E=\left\{\xi \in \mathbb{R}^{m \times n}:\left\langle\xi_{i} ; \xi_{j}\right\rangle=r_{i} r_{j} \delta_{i j}\right\}
$$

where $\delta_{i j}$ is the Kronecker symbol. Let

$$
A=\operatorname{diag}\left(\frac{1}{r_{1}}, \ldots, \frac{1}{r_{n}}\right) \in \mathbb{R}^{n \times n}
$$

then

$$
\begin{aligned}
E & =\left\{\xi \in \mathbb{R}^{m \times n}: \lambda_{i}(\xi A)=1, i=1, \ldots, n\right\} \\
\operatorname{Rco} E & =\operatorname{co} E=\left\{\xi \in \mathbb{R}^{m \times n}: \lambda_{n}(\xi A) \leq 1\right\} .
\end{aligned}
$$

Remark 5.2. (1) The first case with $m=2$ will apply to the complex eikonal equation.

(2) If we consider the second case with $m=3, n=2, r_{1}=r_{2}$ and

$$
\xi=D u(x, y)=\left(\begin{array}{cc}
u_{x}^{1} & u_{y}^{1} \\
u_{x}^{2} & u_{y}^{2} \\
u_{x}^{3} & u_{y}^{3}
\end{array}\right)
$$

then $D u \in E$ means, in geometrical terms, that the surface has been parametrized globally by isothermal coordinates. 
(3) The case $m=n=2$ has been established in [6]. Recently Bousselsal and Le Dret [1] (still when $m=n=2$ ), in the context of nonlinear elasticity, found that (c.f. their Theorem 3.11 with $\varepsilon=0$ ) if $r^{1}=r^{2}=1$ then

$$
F=\left\{\xi \in \mathbb{R}^{2 \times 2}:\left|\xi^{i}\right|^{2}+\left|\left\langle\xi^{1} ; \xi^{2}\right\rangle\right| \leq 1, \quad i=1,2\right\} \subset \operatorname{Rco} E .
$$

This is of course compatible with the result of [6] and of the above theorem. Note however that $F \neq$ Rco $E$ since, for example,

$$
\xi=\left(\begin{array}{ll}
\frac{1}{\sqrt{3}} & 0 \\
\frac{\sqrt{2}}{\sqrt{3}} & 0
\end{array}\right) \in \operatorname{Rco} E, \xi \notin F .
$$

Proof. Obviously the two cases are transposed one from each other and we therefore will only deal with the second one. We start by letting

$$
X=\left\{\xi \in \mathbb{R}^{m \times n}: \lambda_{n}(\xi A) \leq 1\right\} .
$$

Step 1 : We first prove that $\operatorname{Rco} E \subset \operatorname{co} E \subset X$. The first inclusion Rco $E \subset \operatorname{co} E$ always holds and the second one follows from the following two observations.

First we note that the set $X$ is convex since the function $\xi \rightarrow \lambda_{n}(\xi A)$ is convex (c.f., for example, Lemma 7.10 in [6]).

Next observe that the inclusion $E \subset X$ holds. Indeed if $\xi \in E$ (note that $\left.\xi A=\left(\frac{\xi_{1}}{r_{1}}, \ldots, \frac{\xi_{n}}{r_{n}}\right)\right)$ then

$$
\begin{aligned}
\xi & \in E \Leftrightarrow\left\langle(\xi A)_{i} ;(\xi A)_{j}\right\rangle=\delta_{i j} \Leftrightarrow \\
\xi A & \in O(m, n) \Leftrightarrow \lambda_{\alpha}(\xi A)=1,1 \leq \alpha \leq n .
\end{aligned}
$$

Step 2 : We now discuss the reverse inclusions $X \subset \operatorname{Rco} E \subset$ co $E$. Let $\xi \in X$. Replacing $\xi$ by $\xi A$ we can assume, without loss of generality, that $A=I_{n \times n}$. Applying Theorem 12.4, we can find $R \in O(n)$ such that

$$
\xi R=\widetilde{\xi}=\left(\widetilde{\xi}_{1}, \ldots, \widetilde{\xi}_{n}\right), \text { with }\left\langle\widetilde{\xi}_{i} ; \widetilde{\xi}_{j}\right\rangle=\left|\widetilde{\xi}_{i}\right|\left|\widetilde{\xi}_{j}\right| \delta_{i j}, \lambda_{i}(\xi)=\left|\widetilde{\xi}_{i}\right| .
$$

Since the sets $E$ and $X$ are invariant under the (right) action of $O(n)$ we will assume, without loss of generality, that

$$
\xi=\left(\xi_{1}, \ldots, \xi_{n}\right), \text { with }\left\langle\xi_{i} ; \xi_{j}\right\rangle=0, \forall i \neq j, \lambda_{i}(\xi)=\left|\xi_{i}\right| \leq 1,1 \leq i \leq n .
$$

It is therefore sufficient to show that such $\xi$ belongs to Rco $E$. 
Assume first that $\left|\xi_{i}\right|>0, \forall i=1, \ldots, n$ and write

$$
\begin{aligned}
\xi & =\left(\xi_{1}, \ldots, \xi_{n}\right)=\frac{1+\left|\xi_{1}\right|}{2} \eta^{+}+\frac{1-\left|\xi_{1}\right|}{2} \eta^{-} \\
& =\frac{1+\left|\xi_{1}\right|}{2}\left(\frac{\xi_{1}}{\left|\xi_{1}\right|}, \xi_{2}, \ldots, \xi_{n}\right)+\frac{1-\left|\xi_{1}\right|}{2}\left(\frac{-\xi_{1}}{\left|\xi_{1}\right|}, \xi_{2}, \ldots, \xi_{n}\right) .
\end{aligned}
$$

Note that $\operatorname{rank}\left\{\eta^{+}-\eta^{-}\right\}=1$ and that if $\eta^{ \pm}=\left(\eta_{1}^{ \pm}, \eta_{2}^{ \pm}, \ldots, \eta_{n}^{ \pm}\right)$then

$$
\left\langle\eta_{i}^{ \pm} ; \eta_{j}^{ \pm}\right\rangle=0, \forall i \neq j,\left|\eta_{1}^{ \pm}\right|=1,\left|\eta_{i}^{ \pm}\right| \leq 1, \forall i=2, \ldots, n .
$$

Iterating the procedure with the second component and then with the other ones we conclude that $\xi$ can be written as a rank one convex combination of elements of $E$, i.e. $\xi \in \operatorname{Rco} E$.

The case where some of the $\left|\xi_{i}\right|=0$ is handled similarly. For example if $\xi_{1}=0$ we write

$$
\begin{aligned}
\xi & =\left(\xi_{1}, \ldots, \xi_{n}\right)=\frac{1}{2} \eta^{+}+\frac{1}{2} \eta^{-} \\
& =\frac{1}{2}\left(e_{1}, \xi_{2}, \ldots, \xi_{n}\right)+\frac{1}{2}\left(-e_{1}, \xi_{2}, \ldots, \xi_{n}\right)
\end{aligned}
$$

where $e_{1}$ is any vector of $\mathbb{R}^{m}$ such that

$$
\left|e_{1}\right|=1,\left\langle\xi_{i} ; e_{1}\right\rangle=0, \forall i=2, \ldots, n .
$$

Iterate again the procedure to deduce that $\xi \in \operatorname{Rco} E$, as claimed. This concludes the proof.

We can finally apply the results in Section 2 to obtain the following existence theorem for the complex eikonal equation (the case $n=2$ is already in [6]).

Corollary 5.3. Let $\Omega \subset \mathbb{R}^{n}$ be a bounded open set, $f: \Omega \times \mathbb{R} \times \mathbb{R} \rightarrow \mathbb{R}$, $f=f(x, u, v)$, a continuous function and $\varphi \in W^{1, \infty}(\Omega ; \mathbb{C})$. Then there exists $w \in W^{1, \infty}(\Omega ; \mathbb{C})$ satisfying

$$
\left\{\begin{array}{l}
\sum_{i=1}^{n} w_{x_{i}}^{2}+f^{2}=0, \quad \text { a.e. in } \Omega \\
w=\varphi, \quad \text { on } \partial \Omega
\end{array}\right.
$$

where $w_{x_{i}}=\partial w / \partial x_{i}$. Or, in other words, there exists $(u, v) \in W^{1, \infty}\left(\Omega ; \mathbb{R}^{2}\right)$ such that

$$
\left\{\begin{array}{l}
|D v|^{2}=|D u|^{2}+f^{2}, \quad \text { a.e. in } \Omega, \\
\langle D v ; D u\rangle=0, \quad \text { a.e. in } \Omega, \\
(u, v)=\left(\varphi_{1}, \varphi_{2}\right), \quad \text { on } \partial \Omega .
\end{array}\right.
$$


Proof. In fact we solve a more restrictive problem of the type of the above theorem, i.e.

$$
\left\{\begin{array}{l}
|D u|^{2}=r^{2}, \quad \text { a.e. in } \Omega, \\
|D v|^{2}=r^{2}+f^{2}, \quad \text { a.e. in } \Omega, \\
\langle D v ; D u\rangle=0, \quad \text { a.e. in } \Omega \\
(u, v)=\left(\varphi_{1}, \varphi_{2}\right), \quad \text { on } \partial \Omega
\end{array}\right.
$$

where $r>0$ is chosen so large that

$$
\lambda_{2}(A D \varphi) \leq 1-\varepsilon
$$

for $\varepsilon>0$, i.e.

$$
D \varphi(x) \text { compactly contained in } \operatorname{int} \operatorname{Rco} E \text {, a.e. } x \in \Omega \text {. }
$$

We may then apply Corollary 2.9 with $F_{1}=\lambda_{1}+\lambda_{2}-2, F_{2}=\lambda_{2}-1$ (in case $f$ is constant, otherwise proceed as in [6]).

\section{A problem of optimal Design}

We will denote, in this section, the set of $2 \times 2$ symmetric matrices by $\mathbb{R}_{s}^{2 \times 2}$. Our algebraic result is as follows.

Theorem 6.1. Let

$$
E=\left\{\xi \in \mathbb{R}_{s}^{2 \times 2}: \text { trace } \xi \in\{0,1\}, \operatorname{det} \xi \geq 0\right\},
$$

then

$$
\begin{aligned}
\operatorname{Rco} E & =\operatorname{co} E=\left\{\xi \in \mathbb{R}_{s}^{2 \times 2}: 0 \leq \operatorname{trace} \xi \leq 1, \operatorname{det} \xi \geq 0\right\}, \\
\operatorname{int} \operatorname{Rco} E & =\left\{\xi \in \mathbb{R}_{s}^{2 \times 2}: 0<\operatorname{trace} \xi<1, \operatorname{det} \xi>0\right\} .
\end{aligned}
$$

Remark 6.2. Note that it is slightly surprising that the rank one convex hull is in fact convex since the function $\xi \rightarrow \operatorname{det} \xi$ is not convex.

Proof. We call

$$
\begin{aligned}
& X=\left\{\xi \in \mathbb{R}_{s}^{2 \times 2}: 0 \leq \operatorname{trace} \xi \leq 1, \operatorname{det} \xi \geq 0\right\} \\
& Y=\left\{\xi \in \mathbb{R}_{s}^{2 \times 2}: 0<\operatorname{trace} \xi<1, \operatorname{det} \xi>0\right\} .
\end{aligned}
$$

Step 1: we first prove that

$$
\text { Rco } E \subset \operatorname{co} E \subset X \text {. }
$$

The first inclusion always holds and the second one follows from the fact that $E \subset X$ and that $X$ is convex. Indeed let $\xi, \eta \in X, 0 \leq t \leq 1$ we wish to show that $t \xi+(1-t) \eta \in X$. It is clear that the first inequality in the definition of $X$ holds since $\xi \rightarrow$ trace $\xi$ is linear. We now show the second one. Observe first that since $\operatorname{det} \xi=\xi_{11} \xi_{22}-\xi_{12}^{2}$, $\operatorname{det} \eta=\eta_{11} \eta_{22}-\eta_{12}^{2} \geq 0$ and trace $\xi$, trace $\eta \geq 0$ then $\xi_{11}, \xi_{22}, \eta_{11}, \eta_{22} \geq$ 
0 and we therefore have (assume below that $\xi_{11}, \eta_{11}>0$ otherwise the inequality below is trivial)

$$
\begin{aligned}
\langle\widetilde{\xi} ; \eta\rangle & \equiv \xi_{11} \eta_{22}+\eta_{11} \xi_{22}-2 \xi_{12} \eta_{12} \\
& \geq \xi_{11} \frac{\eta_{12}^{2}}{\eta_{11}}+\eta_{11} \frac{\xi_{12}^{2}}{\xi_{11}}-2 \xi_{12} \eta_{12}=\frac{\left(\xi_{11} \eta_{12}-\eta_{11} \xi_{12}\right)^{2}}{\xi_{11} \eta_{11}} \geq 0 .
\end{aligned}
$$

We therefore deduce that

$$
\operatorname{det}(t \xi+(1-t) \eta)=t^{2} \operatorname{det} \xi+t(1-t)\langle\widetilde{\xi} ; \eta\rangle+(1-t)^{2} \operatorname{det} \eta \geq 0 \text {. }
$$

Step 2 : we now prove that

$$
X \subset \operatorname{Rco} E \text {. }
$$

Since $X$ is compact, as usual, it is enough to prove that $\partial X \subset \operatorname{Rco} E$. However it is easy to see that

$$
\partial X=E \cup\left\{\xi \in \mathbb{R}_{s}^{2 \times 2}: 0 \leq \operatorname{trace} \xi \leq 1, \operatorname{det} \xi=0\right\}
$$

and therefore the proof will be completed once we will show that the second set in the right hand side is in Rco $E$. Assume that $\xi$ is such that $0<t=\operatorname{trace} \xi<1$ and $\operatorname{det} \xi=0$. We can then write

$$
\begin{aligned}
\xi & =\left(\begin{array}{ll}
x & \sqrt{x(t-x)} \\
\sqrt{x(t-x)} & t-x
\end{array}\right)=t \xi_{1}+(1-t) \xi_{2} \\
& =t\left(\begin{array}{ll}
\alpha & \sqrt{\alpha(1-\alpha)} \\
\sqrt{\alpha(1-\alpha)} & 1-\alpha
\end{array}\right)+(1-t)\left(\begin{array}{ll}
0 & 0 \\
0 & 0
\end{array}\right)
\end{aligned}
$$

where $x=t \alpha$. The result follows from the facts that $\xi_{1}, \xi_{2} \in E$ and $\operatorname{det}\left(\xi_{1}-\xi_{2}\right)=0$.

Step 3 : The fact that $Y=\operatorname{int} \operatorname{Rco} E$ is easy.

Combining the above theorem with Corollary 2.9 we get

Theorem 6.3. Let $\Omega \subset \mathbb{R}^{2}$ be an open set and $\varphi \in C_{\text {piec }}^{2}(\bar{\Omega})$ satisfy

$$
\left\{\begin{array}{l}
0 \leq \Delta \varphi(x) \leq 1, \quad \text { a.e. } x \in \Omega, \\
\operatorname{det} D^{2} \varphi(x)>0, \quad \text { a.e. } x \in \Omega,
\end{array}\right.
$$

or $\varphi \in W^{2, \infty}(\Omega)$ such that

$$
\left\{\begin{array}{l}
\varepsilon \leq \Delta \varphi(x) \leq 1-\varepsilon, \quad \text { a.e. } x \in \Omega \\
\operatorname{det} D^{2} \varphi(x) \geq \varepsilon, \quad \text { a.e. } x \in \Omega
\end{array}\right.
$$

for some $\varepsilon>0$; then there exists $w \in \varphi+W_{0}^{2, \infty}(\Omega)$

$$
\begin{cases}\Delta w(x) \in\{0,1\}, & \text { a.e. } x \in \Omega, \\ \operatorname{det} D^{2} w(x) \geq 0, & \text { a.e. } x \in \Omega .\end{cases}
$$


Remark 6.4. The above theorem has been proved (except the case with $W^{2, \infty}$ boundary data) in Theorem 3.12 in [6] using the method of confocal ellipses of Murat-Tartar. However the proof we have here (c.f. also [7]) relies on the abstract existence result of Section 2 and on the algebraic theorem above. Of course the use of the abstract theorem is more flexible, because we could imagine, for example, to replace 1 by a function $a(x, u, D u)$, which is out of reach by the explicit method.

\section{A first ACADEMiC EXAMPle}

Inspired by the two preceding examples we look to the problem (recalling that we denote the singular values of a matrix $\xi \in \mathbb{R}^{2 \times 2}$ by $\left.0 \leq \lambda_{1}(\xi) \leq \lambda_{2}(\xi)\right)$.

Theorem 7.1. Let $0 \leq \alpha, \beta<1$ and

$$
E=\left\{\xi \in \mathbb{R}^{2 \times 2}: \lambda_{1}(\xi)+\lambda_{2}(\xi)=1-\alpha, \quad \operatorname{det} \xi \geq \beta\right\}
$$

then

$$
\begin{aligned}
\operatorname{Rco} E & =\left\{\xi \in \mathbb{R}^{2 \times 2}: \lambda_{1}(\xi)+\lambda_{2}(\xi) \leq 1-\alpha, \quad \operatorname{det} \xi \geq \beta\right\} \\
\operatorname{int} \operatorname{Rco} E & =\left\{\xi \in \mathbb{R}^{2 \times 2}: \lambda_{1}(\xi)+\lambda_{2}(\xi)<1-\alpha, \quad \operatorname{det} \xi>\beta\right\} .
\end{aligned}
$$

Remark 7.2. Note that if $(1-\alpha)^{2}-4 \beta<0$ then $E=\emptyset$. It can also be proved that if $f(\xi)=\lambda_{1}(\xi)+\lambda_{2}(\xi)$ and $g(\xi)=|\xi|^{2}-2 \operatorname{det} \xi$ then

$$
\operatorname{co} E=\left\{\xi \in \mathbb{R}^{2 \times 2}: f(\xi) \leq 1-\alpha, g(\xi) \leq(1-\alpha)^{2}-4 \beta\right\},
$$

which in the case $\beta=0$ takes the simpler form

$$
\text { co } E=\left\{\xi \in \mathbb{R}^{2 \times 2}: \lambda_{1}(\xi)+\lambda_{2}(\xi) \leq 1-\alpha\right\} \text {. }
$$

Proof. We let

$$
X=\left\{\xi \in \mathbb{R}^{2 \times 2}: \lambda_{1}(\xi)+\lambda_{2}(\xi) \leq 1-\alpha, \quad \operatorname{det} \xi \geq \beta\right\} .
$$

The fact that Rco $E \subset X$ is elementary since $E \subset X$ and the functions $\xi \rightarrow \lambda_{1}(\xi)+\lambda_{2}(\xi)$ and $\xi \rightarrow-\operatorname{det} \xi$ are polyconvex. So we only need to show the converse inclusion. The compactness of $X$ implies that the result will be proved if we can show that $\partial X \subset$ Rco $E$. Since

$$
\partial X=E \cup\left\{\xi \in \mathbb{R}^{2 \times 2}: \lambda_{1}(\xi)+\lambda_{2}(\xi) \leq 1-\alpha, \quad \operatorname{det} \xi=\beta\right\},
$$

we only need to show that any $\xi \in \mathbb{R}^{2 \times 2}$ with $\lambda_{1}(\xi)+\lambda_{2}(\xi)<1-\alpha$ and $\operatorname{det} \xi=\beta$ belongs to Rco $E$. Choose $\eta \in \mathbb{R}^{2 \times 2}$ be any matrix of rank one such that

$$
\langle\widetilde{\xi} ; \eta\rangle \equiv \xi_{11} \eta_{22}+\eta_{11} \xi_{22}-\xi_{12} \eta_{21}-\xi_{21} \eta_{12}=0
$$

Define then for $t \in \mathbb{R}$

$$
\xi_{t}=\xi+t \eta
$$


and observe that by construction $\operatorname{det} \xi_{t}=\operatorname{det} \xi=\beta$. Using again the compactness argument we can find $t_{1}<0<t_{2}$ such that $\xi_{t_{1}}, \xi_{t_{2}} \in E$, i.e.

$$
\lambda_{1}\left(\xi_{t_{i}}\right)+\lambda_{2}\left(\xi_{t_{i}}\right)=1-\alpha, i=1,2
$$

the result then follows at once (the representation formula for int Rco $E$ is easily deduced).

As a corollary we obtain

Corollary 7.3. Let $\Omega \subset \mathbb{R}^{2}$ be an open set and $\varphi \in C_{\text {piec }}^{1}(\bar{\Omega})$ be such that

$$
\lambda_{1}(D \varphi)+\lambda_{2}(D \varphi)<1, \quad \operatorname{det} D \varphi>0, \text { a.e. in } \Omega .
$$

Then there exists $u \in \varphi+W_{0}^{1, \infty}(\Omega)$ satisfying

$$
\lambda_{1}(D u)+\lambda_{2}(D u)=1, \quad \operatorname{det} D u>0, \text { a.e. in } \Omega .
$$

Proof. This theorem follows either directly from Theorem 2.4 or via the approximation property as done below. First find $\delta_{0}>0$ so that

$$
\operatorname{det} D \varphi \geq \delta_{0}>0 \text {. }
$$

Let $\delta \geq \delta_{0}$ and

$$
E_{\delta}=\left\{\xi \in \mathbb{R}^{2 \times 2}: \lambda_{1}(\xi)+\lambda_{2}(\xi)=1-\delta, \quad \operatorname{det} \xi \geq \delta\right\}
$$

$\left(E=E_{\delta_{0}}\right)$ hence according to the above theorem we have

$$
\text { Rco } E_{\delta}=\left\{\xi \in \mathbb{R}^{2 \times 2}: \lambda_{1}(\xi)+\lambda_{2}(\xi) \leq 1-\delta, \quad \operatorname{det} \xi \geq \delta\right\} .
$$

We may then combine Theorem 2.2 and Theorem 2.8 to get the result

\section{A SECOND ACADEMIC EXAMPLE}

We will now consider Example 1.4 and we will compute its rank one convex hull. Recall that the set of $2 \times 2$ symmetric matrices is denoted by $\mathbb{R}_{s}^{2 \times 2}$.

Theorem 8.1. Let $a_{i j}>0, i, j=1,2$ with $a_{12}=a_{21}$. Let

$$
E=\left\{\xi=\left(\xi_{i j}\right) \in \mathbb{R}_{s}^{2 \times 2}:\left|\xi_{i j}\right|=a_{i j}, \quad i, j=1,2\right\} .
$$

then

$$
\operatorname{co} E=\left\{\xi=\left(\xi_{i j}\right) \in \mathbb{R}_{s}^{2 \times 2}:\left|\xi_{i j}\right| \leq a_{i j}, \quad i, j=1,2\right\} .
$$

Case 1: If $a_{11} a_{22}-a_{12}^{2}<0$ then

$$
\operatorname{Rco} E=\left\{\xi=\left(\xi_{i j}\right) \in \mathbb{R}_{s}^{2 \times 2}:\left|\xi_{12}\right|=a_{12},\left|\xi_{11}\right| \leq a_{11},\left|\xi_{22}\right| \leq a_{22}\right\} \text {. }
$$


Case 2: If $a_{11} a_{22}-a_{12}^{2}=0$ then

$$
\operatorname{Rco} E=\left\{\begin{array}{l}
\xi=\left(\xi_{i j}\right) \in \mathbb{R}_{s}^{2 \times 2}:\left|\xi_{i j}\right| \leq a_{i j}, \quad i, j=1,2 \\
\left|a_{22} \xi_{11}-a_{11} \xi_{22}\right| \leq-\operatorname{det} \xi=-\xi_{11} \xi_{22}+\xi_{12}^{2}
\end{array}\right\} \text {. }
$$

Case 3: If $a_{11} a_{22}-a_{12}^{2}>0$ then

$$
\operatorname{Rco} E \subset\left\{\begin{array}{c}
\xi=\left(\xi_{i j}\right) \in \mathbb{R}_{s}^{2 \times 2}:\left|\xi_{i j}\right| \leq a_{i j}, \quad i, j=1,2 \\
\left|a_{22} \xi_{11}-a_{11} \xi_{22}\right| \leq a_{11} a_{22}-a_{12}^{2}-\operatorname{det} \xi
\end{array}\right\} \text {. }
$$

Remark 8.2. (1) If we consider the $2 \times 2$ matrix 0 then it is clear that in Case 1: $0 \notin \mathrm{Rco} E$, while in Case $2: 0 \in \operatorname{Rco} E$ but $0 \notin$ int Rco $E$. It can be shown, however, that in Case $3: 0 \in$ int Rco $E$.

(2) To apply the above theorem to partial differential equations one needs that int Rco $E \neq \emptyset$; this does not happen in Case 1, contrary to the two other cases. However we need also the approximation property (c.f. Definition 2.7) of the rank one convex hull that we are not able, at the moment, to prove.

(3) In Case 3 we were not able to find a complete characterization of Rco E; the set given in the right hand side of the inclusion is too large.

Proof. The representation formula for the convex hull is trivial.

Case 1 : We denote by

$$
X=\left\{\xi=\left(\xi_{i j}\right) \in \mathbb{R}_{s}^{2 \times 2}:\left|\xi_{12}\right|=a_{12},\left|\xi_{11}\right| \leq a_{11},\left|\xi_{22}\right| \leq a_{22}\right\} .
$$

1 ) It is clear that $X \subset \operatorname{Rco} E$. Indeed write any $\xi \in X$ (assume without loss of generality that $\xi_{12}=a_{12}$ ) as

$\xi=\left(\begin{array}{ll}\xi_{11} & a_{12} \\ a_{12} & \xi_{22}\end{array}\right)=\frac{a_{11}+\xi_{11}}{2 a_{11}}\left(\begin{array}{ll}a_{11} & a_{12} \\ a_{12} & \xi_{22}\end{array}\right)+\frac{a_{11}-\xi_{11}}{2 a_{11}}\left(\begin{array}{ll}-a_{11} & a_{12} \\ a_{12} & \xi_{22}\end{array}\right)$

and similarly

$\left(\begin{array}{ll} \pm a_{11} & a_{12} \\ a_{12} & \xi_{22}\end{array}\right)=\frac{a_{22}+\xi_{22}}{2 a_{22}}\left(\begin{array}{ll} \pm a_{11} & a_{12} \\ a_{12} & a_{22}\end{array}\right)+\frac{a_{22}-\xi_{22}}{2 a_{22}}\left(\begin{array}{ll} \pm a_{11} & a_{12} \\ a_{12} & -a_{22}\end{array}\right)$

to deduce that $\xi \in \operatorname{Rco} E$.

2) We now show the reverse inclusion. Observe first that trivially $E \subset X$. Therefore to get the claimed result it is enough to show that $X$ is a rank one convex set. So let $\xi, \eta \in X$ with $\operatorname{det}(\xi-\eta)=0$ and $0<t<1$. Note that since $\xi, \eta \in X$ then $\left(\xi_{12}-\eta_{12}\right)^{2}$ is either 0 or $4 a_{12}^{2}$. The second case cannot happen since we would have

$$
\left\{\begin{array}{c}
0=\operatorname{det}(\xi-\eta)=\left(\xi_{11}-\eta_{11}\right)\left(\xi_{22}-\eta_{22}\right)-\left(\xi_{12}-\eta_{12}\right)^{2} \\
\leq\left(\left|\xi_{11}\right|+\left|\eta_{11}\right|\right)\left(\left|\xi_{22}\right|+\left|\eta_{22}\right|\right)-4 a_{12}^{2} \leq 4 a_{11} a_{22}-4 a_{12}^{2}<0
\end{array},\right.
$$

which is absurd. So the only case that can happen is $\xi_{12}=\eta_{12}$ (with $\left.\left|\xi_{12}\right|=a_{12}\right)$. The claimed result $t \xi+(1-t) \eta \in X$ is then immediate. 
Case 2: As before we call

$$
X=\left\{\begin{array}{l}
\xi=\left(\xi_{i j}\right) \in \mathbb{R}_{s}^{2 \times 2}:\left|\xi_{i j}\right| \leq a_{i j}, \quad i, j=1,2 \\
\left|a_{22} \xi_{11}-a_{11} \xi_{22}\right| \leq-\operatorname{det} \xi=-\xi_{11} \xi_{22}+\xi_{12}^{2}
\end{array}\right\} .
$$

1) We easily see that $E \subset X$ and that $X$ is a rank one convex (in fact even polyconvex) set since all the functions involved with the inequalities are polyconvex and thus rank one convex. We therefore have Rco $E \subset X$.

2) We now discuss the inclusion $X \subset$ Rco $E$. We start by observing that if we can show (c.f. below) that $\partial X \subset$ Rco $E$ then the result will follow. Indeed if $\xi \in \operatorname{int} X$, since $X$ is compact, we can find for every $\lambda \in \mathbb{R}_{s}^{2 \times 2}$ with rank $\lambda=1, t_{1}<0<t_{2}$, such that

$$
\xi+t_{1} \lambda, \xi+t_{2} \lambda \in \partial X
$$

and hence since $\partial X \subset \operatorname{Rco} E$ we get that $\xi \in \operatorname{Rco} E$.

We now wish to show that if $\xi \in \partial X$ then $\xi \in \operatorname{Rco} E$. Note first that the last inequality in the definition of $X$ is equivalent, bearing in mind that $a_{11} a_{22}-a_{12}^{2}=0$, to

$$
\left\{\begin{array}{l}
0 \leq a_{12}^{2}-\xi_{12}^{2} \leq\left(a_{11}-\xi_{11}\right)\left(a_{22}+\xi_{22}\right) \\
0 \leq a_{12}^{2}-\xi_{12}^{2} \leq\left(a_{11}+\xi_{11}\right)\left(a_{22}-\xi_{22}\right)
\end{array} .\right.
$$

Observe that if either $\left|\xi_{11}\right|=a_{11}$ or $\left|\xi_{22}\right|=a_{22}$ then by (12) necessarily $\left|\xi_{12}\right|=a_{12}$. However if $\left|\xi_{12}\right|=a_{12}$ then, by the same argument as in Case 1, we deduce that $\xi \in \operatorname{Rco} E$.

So we now assume that $\left|\xi_{i j}\right|<a_{i j}$ and (since $\xi \in \partial X$ ) one of the inequalities in (12) is an equality and without loss of generality say the first one while the second one is a strict inequality. If we call

$$
\begin{aligned}
& V_{1}=\left\{\xi=\left(\xi_{i j}\right) \in \mathbb{R}_{s}^{2 \times 2}: a_{12}^{2}-\xi_{12}^{2}=\left(a_{11}-\xi_{11}\right)\left(a_{22}+\xi_{22}\right)\right\} \\
& V_{2}=\left\{\xi=\left(\xi_{i j}\right) \in \mathbb{R}_{s}^{2 \times 2}: a_{12}^{2}-\xi_{12}^{2}=\left(a_{11}+\xi_{11}\right)\left(a_{22}-\xi_{22}\right)\right\} \\
& Y_{1}=\partial X \cap V_{1}
\end{aligned}
$$

then $\xi \in \operatorname{relint} Y_{1}$ (the relative interior of $Y_{1}$ ). We can then choose

$$
\lambda=\left(\begin{array}{ll}
\lambda_{1}^{2} & \lambda_{1} \lambda_{2} \\
\lambda_{1} \lambda_{2} & \lambda_{2}^{2}
\end{array}\right)
$$

with $\lambda_{1}, \lambda_{2} \neq 0$ so that

$$
\xi+t \lambda \in V_{1}, \forall t \in \mathbb{R}
$$

this is always possible by choosing

$$
\lambda_{1}=1, \lambda_{2}=-\frac{a_{12}+\xi_{12}}{a_{11}-\xi_{11}}
$$


or more generally any non zero solution of

$$
\lambda_{2}^{2}\left(a_{11}-\xi_{11}\right)+2 \lambda_{1} \lambda_{2} \xi_{12}-\lambda_{1}^{2}\left(a_{22}+\xi_{22}\right)=0 .
$$

Then since $\xi \in \operatorname{relint} Y_{1}$ and $Y_{1}$ is compact we can find $t_{1}<0<t_{2}$, such that

$$
\xi+t_{1} \lambda, \xi+t_{2} \lambda \in \partial Y_{1} .
$$

But $\widetilde{\xi} \in \partial Y_{1}$ means that either $\left|\widetilde{\xi}_{i j}\right|=a_{i j}$ for a certain $i, j$ and this case has already been dealt with or $\widetilde{\xi} \in V_{2}$. Hence the only case that requires still to be analyzed is when $\left|\xi_{i j}\right|<a_{i j}$ and $\xi \in V_{1} \cap V_{2}$, i.e. when in (12) the two inequalities are actually equalities. Note that any $\xi \in V_{1} \cap V_{2}$ is of the form

$$
\xi=\xi_{11}\left(\begin{array}{ll}
1 & \pm \sqrt{\frac{a_{22}}{a_{11}}} \\
\pm \sqrt{\frac{a_{22}}{a_{11}}} & \frac{a_{22}}{a_{11}}
\end{array}\right)
$$

and thus if we denote by

$$
\lambda=\left(\begin{array}{ll}
1 & \pm \sqrt{\frac{a_{22}}{a_{11}}} \\
\pm \sqrt{\frac{a_{22}}{a_{11}}} & \frac{a_{22}}{a_{11}}
\end{array}\right),
$$

which is a matrix of rank one, we find that

$$
\xi+t \lambda \in V_{1} \cap V_{2}, \forall t \in \mathbb{R} .
$$

The usual argument then applies, namely if $\left|\xi_{i j}\right|<a_{i j}$ and $\xi \in V_{1} \cap V_{2}$ we can find $t_{1}<0<t_{2}$, such that one of the inequalities $\left|\xi_{i j}\right|<a_{i j}$ becomes an equality; in which case we conclude that $\xi \in \operatorname{Rco} E$ by the previous steps.

Case 3 : The claimed inclusion follows for the same reasons as in Case 2.

\section{The case of potential wells}

We now discuss how to apply Theorem 3.2 to the case of two potential wells under incompressibility constraint (this result has recently been obtained by Müller-Sverak [14] and we show here how our method gives the same result). We start, as usual, with some algebraic considerations.

Proposition 9.1. Let $0<\lambda<1$,

$$
\Lambda=\left(\begin{array}{ll}
\lambda & 0 \\
0 & 1 / \lambda
\end{array}\right)
$$


and

$$
E=S O(2) I \cup S O(2) \Lambda
$$

Let

$$
\begin{aligned}
& F(\xi)=\sqrt{\left(\xi_{11}-\xi_{22}\right)^{2}+\left(\xi_{12}+\xi_{21}\right)^{2}}+\sqrt{\left(\frac{1}{\lambda} \xi_{11}-\lambda \xi_{22}\right)^{2}+\left(\lambda \xi_{12}+\frac{1}{\lambda} \xi_{21}\right)^{2}} \\
& G(\xi)=\left(\xi_{11}-\xi_{22}\right)^{2}+\left(\xi_{12}+\xi_{21}\right)^{2}+\left(\frac{1}{\lambda} \xi_{11}-\lambda \xi_{22}\right)^{2}+\left(\lambda \xi_{12}+\frac{1}{\lambda} \xi_{21}\right)^{2} .
\end{aligned}
$$

Then $F$ and $G$ are convex and invariant under the (left) action of $S O(2)$. Moreover

$$
\begin{gathered}
E=\left\{\xi \in \mathbb{R}^{2 \times 2}: F(\xi)=\frac{1}{\lambda}-\lambda, G(\xi)=\left(\frac{1}{\lambda}-\lambda\right)^{2}, \operatorname{det} \xi=1\right\} \\
\operatorname{Rco} E=\left\{\xi \in \mathbb{R}^{2 \times 2}: F(\xi) \leq \frac{1}{\lambda}-\lambda, \operatorname{det} \xi=1\right\} \\
\operatorname{int} \operatorname{Rco} E=\left\{\xi \in \mathbb{R}^{2 \times 2}: F(\xi)<\frac{1}{\lambda}-\lambda, \operatorname{det} \xi=1\right\} .
\end{gathered}
$$

Remark 9.2. Note that $\xi_{1}=\operatorname{diag}(1-\delta, 1 /(1-\delta)) \in \operatorname{int} \operatorname{Rco} E$ and $\xi_{2}=\operatorname{diag}(\lambda /(1-\delta),(1-\delta) / \lambda) \in \operatorname{int} \operatorname{Rco} E$ for $\delta>0$ sufficiently small.

Proof. The result follows from the representation obtained by Sverak and Corollary 8.3 of [6].

1) The fact that $F$ and $G$ are convex and invariant under the (left) action of $S O(2)$ is easy.

2) Let us show now that if

$$
X=\left\{\xi \in \mathbb{R}^{2 \times 2}: F(\xi)=\frac{1}{\lambda}-\lambda, G(\xi)=\left(\frac{1}{\lambda}-\lambda\right)^{2}, \operatorname{det} \xi=1\right\}
$$

then $E=X$. The inclusion $E \subset X$ is easy since $F(I)=F(\Lambda)=\frac{1}{\lambda}-\lambda, G(I)=G(\Lambda)=\left(\frac{1}{\lambda}-\lambda\right)^{2}, \operatorname{det} I=\operatorname{det} \Lambda=1$ and $F, G$ and det are invariant under the (left) action of $S O(2)$. We now discuss the reverse inclusion. Let $\xi \in X$ then either

$$
\left[\left(\xi_{11}-\xi_{22}\right)^{2}+\left(\xi_{12}+\xi_{21}\right)^{2}\right]=0
$$

which implies that $\xi \in S O(2)$ or

$$
\left[\left(\frac{1}{\lambda} \xi_{11}-\lambda \xi_{22}\right)^{2}+\left(\lambda \xi_{12}+\frac{1}{\lambda} \xi_{21}\right)^{2}\right]=0
$$


which implies that $\xi \in S O(2) \Lambda$. In either cases we find that $\xi \in E$.

3) Call

$$
Y=\left\{\xi \in \mathbb{R}^{2 \times 2}: F(\xi) \leq \frac{1}{\lambda}-\lambda, \operatorname{det} \xi=1\right\} .
$$

We now show that Rco $E=Y$. To prove this we use the representation formula established by Sverak (c.f. [6]), i.e.,

$\operatorname{Rco} E=\left\{\begin{array}{c}\xi=\left(\begin{array}{ll}y_{1} & -y_{2} \\ y_{2} & y_{1}\end{array}\right)\left(\begin{array}{ll}1 & 0 \\ 0 & 1\end{array}\right)+\left(\begin{array}{ll}z_{1} & -z_{2} \\ z_{2} & z_{1}\end{array}\right)\left(\begin{array}{ll}\lambda & 0 \\ 0 & 1 / \lambda\end{array}\right) \\ \sqrt{y_{1}^{2}+y_{2}^{2}}+\sqrt{z_{1}^{2}+z_{2}^{2}} \leq 1, \operatorname{det} \xi=1\end{array}\right\}$.

Expressing $y_{1}, y_{2}, z_{1}, z_{2}$ in terms of $\xi_{i j}$ we find

$$
\left\{\begin{array} { l } 
{ \xi _ { 1 1 } = y _ { 1 } + \lambda z _ { 1 } } \\
{ \xi _ { 1 2 } = - ( y _ { 2 } + \frac { 1 } { \lambda } z _ { 2 } ) } \\
{ \xi _ { 2 1 } = y _ { 2 } + \lambda z _ { 2 } } \\
{ \xi _ { 2 2 } = y _ { 1 } + \frac { 1 } { \lambda } z _ { 1 } }
\end{array} \Leftrightarrow \left\{\begin{array}{l}
\left(\frac{1}{\lambda}-\lambda\right) y_{1}=\frac{1}{\lambda} \xi_{11}-\lambda \xi_{22} \\
\left(\frac{1}{\lambda}-\lambda\right) y_{2}=\lambda \xi_{12}+\frac{1}{\lambda} \xi_{21} \\
\left(\frac{1}{\lambda}-\lambda\right) z_{1}=-\left(\xi_{11}-\xi_{22}\right) \\
\left(\frac{1}{\lambda}-\lambda\right) z_{2}=-\left(\xi_{12}+\xi_{21}\right)
\end{array}\right.\right.
$$

Since

$$
F(\xi)=\left(\frac{1}{\lambda}-\lambda\right)\left(\sqrt{y_{1}^{2}+y_{2}^{2}}+\sqrt{z_{1}^{2}+z_{2}^{2}}\right)
$$

we get immediately the result.

4) We now discuss the representation formula for int Rco $E$. Call $Z$ the right hand side in the formula. The inclusion $Z \subset \operatorname{int} \operatorname{Rco} E$ is clear and so we show the reverse one. Let $\xi \in \operatorname{int} \operatorname{Rco} E$; then up to a rotation we can always assume that $\xi_{12}=0$ and hence since $\operatorname{det} \xi=1$ we deduce that if

$$
\xi_{t}=\left(\begin{array}{ll}
\xi_{11} & 0 \\
\xi_{21}+t & 1 / \xi_{11}
\end{array}\right)
$$

then $\xi=\xi_{0}$ and

$$
\operatorname{det} \xi_{t} \equiv 1, \forall t \in \mathbb{R}
$$

Since $\xi \in \operatorname{int} \operatorname{Rco} E$ we find that $\xi_{t} \in \operatorname{Rco} E$ for all $t$ small enough. Observe finally that the function $t \rightarrow F\left(\xi_{t}\right)$ is strictly convex (note however that the function $\xi \rightarrow F(\xi)$ is not strictly convex) and therefore if $t \neq 0$ is small enough we have

$$
F(\xi)<\frac{1}{2} F\left(\xi_{-t}\right)+\frac{1}{2} F\left(\xi_{t}\right) \leq \frac{1}{\lambda}-\lambda
$$

which is the claimed result $\xi \in Z$. 
Theorem 9.3. Let $\Omega \subset \mathbb{R}^{n}$ be open and

$$
E=S O(2) A \cup S O(2) B
$$

with $\operatorname{det} A=\operatorname{det} B>0$. Let

$$
\xi \in \operatorname{int} \text { Rco } E
$$

then there exists $u \in W^{1, \infty}\left(\Omega ; \mathbb{R}^{2}\right)$ such that

$$
\left\{\begin{array}{c}
D u(x) \in E, \text { a.e. in } \Omega \\
u(x)=\xi x, \text { on } \partial \Omega .
\end{array}\right.
$$

Remark 9.4. If $\operatorname{det} A \neq \operatorname{det} B$ this result was already obtained by Müller-Sverak [13] and Dacorogna-Marcellini [5].

Proof. Step 1: We start with some algebraic considerations. Observe that there is no loss of generality if we assume that

$$
A=I \text { and } B=\left(\begin{array}{ll}
\lambda & 0 \\
0 & 1 / \lambda
\end{array}\right) .
$$

Indeed first diagonalize $B A^{-1}$, i.e. find $R_{a}, R_{b} \in S O(2)$ so that

$$
R_{a} B A^{-1} R_{b}=\Lambda=\left(\begin{array}{ll}
\lambda & 0 \\
0 & 1 / \lambda
\end{array}\right)
$$

we therefore deduce that

$$
R_{-b} E A^{-1} R_{b}=S O(2) I \cup S O(2) \Lambda .
$$

Step 2: We define for $\delta \in(0,1]$

$$
I_{\delta}=\left(\begin{array}{ll}
1-\delta & 0 \\
0 & \frac{1}{1-\delta}
\end{array}\right), \quad \Lambda_{\delta}=\left(\begin{array}{ll}
\frac{\lambda}{1-\delta} & 0 \\
0 & \frac{1-\delta}{\lambda}
\end{array}\right) .
$$

Observe that $I_{\delta}, \Lambda_{\delta} \in \operatorname{int} \operatorname{Rco} E$ and if

$$
E_{\delta}=S O(2) I_{\delta} \cup S O(2) \Lambda_{\delta}
$$

and accordingly $F_{\delta}, G_{\delta}$, we then have

$$
E_{\delta} \subset \operatorname{int} \operatorname{Rco} E
$$

and

$$
K\left(E_{\delta}\right)=\operatorname{Rco} E_{\delta} \subset \operatorname{int} \operatorname{Rco} E .
$$

Therefore $E$ and Rco $E$ have the approximation property with $K\left(E_{\delta}\right)=$ Rco $E_{\delta}$. Hence combining Proposition 9.1 with Theorem 3.6 and Theorem 3.2 we get the result. 


\section{The CASE OF NEMATiC ElAStomers}

The problem considered here has been introduced by DeSimoneDolzmann [9].

We begin with the computation of the rank one convex hull; this follows from [8] and [6] (in the case $n=2,3$ c.f. [9]).

Theorem 10.1. Let $0 \leq \lambda_{1}(A) \leq \ldots \leq \lambda_{n}(A)$ denote the singular values of a matrix $A \in \mathbb{R}^{n \times n}$ and

$$
E=\left\{A: \lambda_{i}(A)=a_{i}, i=1, \ldots, n, \operatorname{det} A=\prod_{i=1}^{n} a_{i}\right\}
$$

where $0<a_{1} \leq \ldots \leq a_{n}$. The following then holds

Pco $E=\operatorname{Rco} E=\left\{A: \prod_{i=\nu}^{n} \lambda_{i}(A) \leq \prod_{i=\nu}^{n} a_{i}, \nu=2, \ldots, n, \operatorname{det} A=\prod_{i=1}^{n} a_{i}\right\}$.

Moreover if $0<a_{1}<\ldots<a_{n}$ and $\delta$ is sufficiently small so that

$$
0<a_{1}^{\delta}=(1-\delta)^{1-n} a_{1} \leq a_{2}^{\delta}=(1-\delta) a_{2} \leq \ldots \leq a_{n}^{\delta}=(1-\delta) a_{n}
$$

then $E$ and Rco $E$ have the approximation property with $K\left(E_{\delta}\right)=$ Rco $E_{\delta}$, where

$$
E_{\delta}=\left\{A: \lambda_{i}(A)=a_{i}^{\delta}, i=1, \ldots, n, \operatorname{det} A=\prod_{i=1}^{n} a_{i}\right\} .
$$

Remark 10.2. (i) Note that when $n=3$ and $a_{1}=a_{2}=r^{1 / 6}, a_{3}=$ $r^{-1 / 3}, r<1$, we recover the result of DeSimone-Dolzmann, namely

$$
\text { Pco } E=\operatorname{Rco} E=\left\{A \in \mathbb{R}^{3 \times 3}: \lambda_{i}(A) \in\left[r^{1 / 6}, r^{-1 / 3}\right], \operatorname{det} A=1\right\} \text {. }
$$

(ii) The hypothesis that all the $a_{i}$ are different is too strong and can be weakened; it is enough to assume that the $a_{i}$ are not all equal. It is clear also that if all the $a_{i}$ are equal, then int Rco $E=\emptyset$, since then $E=\operatorname{Rco} E$.

Proof. Let us denote by

$$
X=\left\{A \in \mathbb{R}^{n \times n}: \prod_{i=\nu}^{n} \lambda_{i}(A) \leq \prod_{i=\nu}^{n} a_{i}, \nu=2, \ldots, n, \operatorname{det} A=\prod_{i=1}^{n} a_{i}\right\} .
$$

Step 1 : The fact that Rco $E \subset \operatorname{Pco} E \subset X$ is easy, since $E \subset X$ and the functions

$$
A \rightarrow \prod_{i=\nu}^{n} \lambda_{i}(A)-\prod_{i=\nu}^{n} a_{i}, \nu=2, \ldots, n
$$


are polyconvex and $A \rightarrow \operatorname{det} A-\prod_{i=1}^{n} a_{i}$ is quasiaffine.

Step 2 : As usual by compactness of $X$ it is enough to prove that $\partial X \subset$ Rco $E$. We show the result by induction.

(1) $n=1$. This is trivial.

(2) $n \geq 2$. Any $A \in X$ can, without loss of generality, be assumed of the form

$$
A=\left(\begin{array}{lll}
x_{1} & & \\
& \ddots & \\
& & x_{n}
\end{array}\right)
$$

with $0 \leq x_{1} \leq x_{2} \leq \ldots \leq x_{n}, \prod_{i=\nu}^{n} x_{i} \leq \prod_{i=\nu}^{n} a_{i}, \quad \nu=2, \ldots, n$ and $\Pi_{i=1}^{n} x_{i}=\prod_{i=1}^{n} a_{i}$. Since $A \in \partial X$ we deduce that $\Pi_{i=\bar{\nu}}^{n} x_{i}=\prod_{i=\bar{\nu}}^{n} a_{i}$, for a certain $\bar{\nu} \in\{2, \ldots, n\}$. We can then apply the hypothesis of induction to

$$
\left\{x_{1}, \ldots, x_{\bar{\nu}-1}\right\} \text { and }\left\{a_{1}, \ldots, a_{\bar{\nu}-1}\right\}
$$

and to

$$
\left\{x_{\bar{\nu}}, \ldots, x_{n}\right\} \text { and }\left\{a_{\bar{\nu}}, \ldots, a_{n}\right\} \text {. }
$$

Indeed for the second one this follows from the hypotheses

$$
\begin{aligned}
& \prod_{i=\nu}^{n} x_{i} \leq \prod_{i=\nu}^{n} a_{i}, \quad \nu=\bar{\nu}+1, \ldots, n \\
& \prod_{i=\bar{\nu}}^{n} x_{i}=\prod_{i=\bar{\nu}}^{n} a_{i}
\end{aligned}
$$

while (note that if $\bar{\nu}=2$ then necessarily $x_{1}=a_{1}$ and this part is trivial, so we will assume that $\bar{\nu} \geq 3$ ) for the first one we have

$$
\begin{aligned}
\prod_{i=\nu}^{\bar{\nu}-1} x_{i} & =\prod_{i=\nu}^{n} x_{i}\left(\prod_{i=\bar{\nu}}^{n} x_{i}\right)^{-1}=\prod_{i=\nu}^{n} x_{i}\left(\prod_{i=\bar{\nu}}^{n} a_{i}\right)^{-1} \\
& \leq \prod_{i=\nu}^{n} a_{i}\left(\prod_{i=\bar{\nu}}^{n} a_{i}\right)^{-1}=\prod_{i=\nu}^{\bar{\nu}-1} a_{i}, \quad \nu=2, \ldots, \bar{\nu}-1
\end{aligned}
$$

and

$$
\prod_{i=1}^{\bar{\nu}-1} x_{i}=\prod_{i=1}^{\bar{\nu}-1} a_{i}
$$

We can therefore deduce, by hypothesis of induction, that $A \in \operatorname{Rco} E$. 
Step 3: We now observe that the approximation property follows from the fact that (if $0<a_{1}<\ldots<a_{n}$ )

$$
\operatorname{int} \operatorname{Rco} E=\left\{A: \prod_{i=\nu}^{n} \lambda_{i}(A)<\prod_{i=\nu}^{n} a_{i}, \nu=2, \ldots, n, \operatorname{det} A=\prod_{i=1}^{n} a_{i}\right\} \text {. }
$$

The main result is then, adopting the notations of the above theorem (assume here that $\prod_{i=1}^{n} a_{i}=1$ ).

Theorem 10.3. Let $\Omega \subset \mathbb{R}^{n}$ be open, $0<a_{1}<\ldots<a_{n}$ and

$$
E=\left\{A \in \mathbb{R}^{n \times n}: \lambda_{i}(A)=a_{i}, i=1, \ldots, n, \operatorname{det} A=\prod_{i=1}^{n} a_{i}=1\right\} .
$$

Let $\varphi$ be an affine function $(D \varphi=\xi)$ such that

$$
\begin{array}{r}
\prod_{i=\nu}^{n} \lambda_{i}(\xi)<\prod_{i=\nu}^{n} a_{i}, \nu=2, \ldots, n, \\
\operatorname{det} \xi=1 ;
\end{array}
$$

then there exists (a dense set of) $u \in \varphi+W_{0}^{1, \infty}\left(\Omega ; \mathbb{R}^{n}\right)$ such that

$$
\left\{\begin{array}{l}
\lambda_{i}(D u(x))=a_{i}, i=1,2, \ldots, n, \text { a.e. } x \in \Omega \\
\operatorname{det} D u(x)=1, \text { a.e. } x \in \Omega .
\end{array}\right.
$$

Remark 10.4. Of course a similar result holds if the $a_{i}$ are not constants but depend on $(x, u)$.

Proof. Observe first that

$$
E=\left\{A \in \mathbb{R}^{n \times n}: \prod_{i=\nu}^{n} \lambda_{i}(A)=\prod_{i=\nu}^{n} a_{i}, \nu=2, \ldots, n, \operatorname{det} A=1\right\}
$$

and that $A \rightarrow \prod_{i=\nu}^{n} \lambda_{i}(A), \nu=2, \ldots, n$, are quasiconvex. The result follows then by combining Proposition 10.1 with Theorem 3.2 and Theorem 3.6.

\section{Gauges, Choquet functions and Minkowski theorem FOR POLYCONVEX SETS}

We conclude this article with some general considerations about polyconvex sets. In classical convex analysis the gauge of a convex set, the Choquet function that characterizes extreme points or the Minkowski theorem (often known as Krein-Milman theorem which is its infinite dimensional version) are important tools. We generalize these notions to polyconvex sets. 
We first recall some notations and definitions and we refer to [3] and [6] for more details.

Definition 11.1. (1) For a matrix $A \in \mathbb{R}^{m \times n}$ we let

$$
T(A)=\left(A, a d j_{2} A, \ldots, a d j_{m \wedge n} A\right)
$$

where $a d j_{s} A$ stands for the matrix of all $s \times s$ subdeterminants of the matrix $A, 1 \leq s \leq m \wedge n=\min \{m, n\}$, and

$$
\begin{gathered}
\tau(m, n)=\sum_{s=1}^{m \wedge n}\left(\begin{array}{c}
m \\
s
\end{array}\right)\left(\begin{array}{c}
n \\
s
\end{array}\right), \\
\text { where }\left(\begin{array}{c}
m \\
s
\end{array}\right)=\frac{m !}{s !(m-s) !} .
\end{gathered}
$$

(2) The different envelopes of a given function are defined as

$$
\begin{array}{r}
C f=\sup \{g \leq f: g \text { convex }\}, \\
P f=\sup \{g \leq f: g \text { polyconvex }\}, \\
R f=\sup \{g \leq f: g \text { rank one convex }\},
\end{array}
$$

they are respectively the convex, polyconvex and rank one convex envelope of $f$.

(3) We say that a set $K \subset \mathbb{R}^{m \times n}$ is polyconvex if for every $t_{i} \geq 0$ with $\sum_{i=1}^{\tau(m, n)} t_{i}=1$ and every $A_{i} \in K$ with

$$
\sum_{i=1}^{\tau(m, n)} t_{i} T\left(A_{i}\right)=T\left(\sum_{i=1}^{\tau(m, n)} t_{i} A_{i}\right)
$$

then

$$
\sum_{i=1}^{\tau(m, n)} t_{i} A_{i} \in K
$$

We start with a theorem defining the gauge of a polyconvex set.

Theorem 11.2. Let $K \subset \mathbb{R}^{m \times n}$ be a non empty polyconvex set and let

$$
\chi_{K}(x)=\left\{\begin{array}{c}
0, \text { if } x \in K \\
+\infty, \text { if } x \notin K
\end{array}\right.
$$

be its indicator function. Let $H: \mathbb{R}^{\tau(m, n)} \rightarrow \overline{\mathbb{R}}=\mathbb{R} \cup\{+\infty\}$ be defined as

$$
H(\xi)=\sup _{x \in K}\{\langle T(x) ; \xi\rangle\}
$$

The following then hold

(1) $H$ is lower semicontinuous, convex and positively homogeneous of degree one. 
(2) If $K$ is closed and if $H^{*}: \mathbb{R}^{\tau(m, n)} \rightarrow \overline{\mathbb{R}}=\mathbb{R} \cup\{+\infty\}$ is the conjugate function of $H$ (i.e. $\left.H^{*}\left(\xi^{*}\right)=\sup \left\{\left\langle\xi^{*} ; \xi\right\rangle-H(\xi)\right\}\right)$ then

$$
\begin{aligned}
\chi_{K}(x) & =H^{*}(T(x)) \\
K & =\left\{x \in \mathbb{R}^{m \times n}: H^{*}(T(x)) \leq 0\right\} .
\end{aligned}
$$

(3) If $0 \in K$ then $H(\xi) \geq H(0)=0$. And if $K$ is compact then $H$ takes only finite values.

(4) If $0 \in \operatorname{int} K$ and if $K$ is compact then

$$
H(\xi)=0 \Leftrightarrow \xi=0 ;
$$

and in this case

$$
K=\left\{x \in \mathbb{R}^{m \times n}: H^{0}(T(x)) \leq 1\right\}
$$

where $H^{0}$ is the polar of $H$ (called the gauge of $K$ ), i.e.

$$
H^{0}\left(\xi^{*}\right)=\sup _{\xi \neq 0}\left\{\frac{\left\langle\xi^{*} ; \xi\right\rangle}{H(\xi)}\right\} .
$$

Remark 11.3. (1) When $m=n=2$ we have $H: \mathbb{R}^{2 \times 2} \times \mathbb{R} \rightarrow \overline{\mathbb{R}}=$ $\mathbb{R} \cup\{+\infty\}$ is given by

$$
H(\xi, \delta)=\sup _{x \in K}\{\langle x ; \xi\rangle+\delta \operatorname{det} x\}
$$

and

$$
K=\left\{x \in \mathbb{R}^{2 \times 2}: H^{*}(x, \operatorname{det} x) \leq 0\right\} .
$$

(2) Note that $H^{0}$ is positively homogeneous of degree one but of course this is not the case for the function $x \rightarrow H^{0}(T(x))$.

Example 11.4. Let for $\xi \in \mathbb{R}^{2 \times 2}, 0 \leq \lambda_{1}(\xi) \leq \lambda_{2}(\xi)$ denote its singular values and

$$
K=\left\{\xi \in \mathbb{R}^{2 \times 2}: \lambda_{2}(\xi) \leq a_{2}, \lambda_{1}(\xi) \lambda_{2}(\xi) \leq a_{1} a_{2}\right\},
$$

which is a polyconvex set (c.f. [6]).Then

$$
H^{0}\left(\xi^{*}, \delta^{*}\right)=\max \left\{\frac{\lambda_{2}\left(\xi^{*}\right)}{a_{2}}, \frac{\left|\delta^{*}\right|}{a_{1} a_{2}}\right\}
$$

is a gauge for $K$.

Proof. (1) Since $K$ is non empty then $H>-\infty$. $H$ being the supremum of affine functions, it is convex and lower semicontinuous. The fact that $H$ is positively homogeneous of degree one is easy.

(2) Observe first that according to a result in [3] (c.f. page 199-202) we have

$$
\begin{aligned}
H(\xi) & =\chi_{K}^{p}(\xi) \\
\chi_{K}(x) & =\chi_{K}^{p p}(x)=H^{*}(T(x))
\end{aligned}
$$


hence the result.

(3) This is obvious.

(4) We now show that if $0 \in \operatorname{int} K$ and if $K$ is compact then

$$
H(\xi)=0 \Leftrightarrow \xi=0 .
$$

The implication $(\Leftarrow)$ follows from $(1)$ and we therefore discuss only the converse one. Let $x \in \mathbb{R}^{m \times n}$ be an arbitrary point. Since $0 \in \operatorname{int} K$ we deduce that for every $\varepsilon$ sufficiently small then $\varepsilon x /|x| \in K$ and therefore

$$
0=H(\xi) \geq\left\langle T\left(\frac{\varepsilon x}{|x|}\right) ; \xi\right\rangle
$$

since $x \in \mathbb{R}^{m \times n}$ is arbitrary the above inequality implies that $\xi=0$, as claimed. We prove this last fact only when $m=n=2$, the general case being proved similarly. The inequality (13) reads then (writing $\left.\xi=\left(x^{*}, \delta\right)\right)$

$$
0=H(\xi) \geq \frac{\varepsilon}{|x|}\left\langle x ; x^{*}\right\rangle+\varepsilon^{2} \delta \frac{\operatorname{det} x}{|x|^{2}}, \forall x \in \mathbb{R}^{2 \times 2} .
$$

We therefore get, using the arbitrariness of $\varepsilon$

$$
\left\{\begin{array}{l}
\left\langle x ; x^{*}\right\rangle=0, \forall x \in \mathbb{R}^{2 \times 2} \\
\delta \operatorname{det} x \leq 0, \forall x \in \mathbb{R}^{2 \times 2}
\end{array}\right.
$$

hence $\left(x^{*}, \delta\right)=(0,0)$.

The last identity

$$
K=\left\{x \in \mathbb{R}^{m \times n}: H^{0}(T(x)) \leq 1\right\}
$$

is easy.

The next step is to define a function that characterizes the extreme points. In the convex case this is known as the Choquet function (see for example Pianigiani [15]); but first let us define the following.

Definition 11.5. Let $K \subset \mathbb{R}^{m \times n}$ be polyconvex; we say that $X \in K$ is an extreme point in the polyconvex sense of $K$ if

$$
\left.\begin{array}{c}
T(X)=\sum_{i=1}^{I} t_{i} T\left(A_{i}\right) \\
t_{i}>0 \text { with } \sum_{i=1}^{I} t_{i}=1, A_{i} \in K
\end{array}\right\} \Rightarrow A_{i}=X, i=1, \ldots, I .
$$

The set of extreme points in the polyconvex sense of $K$ is denoted by $K_{e x t}^{p}$. 
Theorem 11.6. Let $K \subset \mathbb{R}^{m \times n}$ be a non empty compact polyconvex set and $K_{e x t}^{p}$ be its extreme points in the polyconvex sense. Then there exists $\varphi: \mathbb{R}^{m \times n} \rightarrow \overline{\mathbb{R}}=\mathbb{R} \cup\{+\infty\}$ a polyconvex function so that

$$
\begin{aligned}
K_{e x t}^{p}= & \{x \in K: \varphi(x)=0\} \\
& \varphi(x) \leq 0 \Leftrightarrow x \in K .
\end{aligned}
$$

Proof. We first define

$$
f(x)=\left\{\begin{array}{c}
-|x|^{2}, \text { if } x \in K \\
+\infty, \text { otherwise }
\end{array}\right.
$$

and

$$
\varphi(x)=\left\{\begin{array}{c}
P f(x)-f(x), \text { if } x \in K \\
+\infty, \text { otherwise } .
\end{array}\right.
$$

In the convex case it is the function $\varphi$ that is the Choquet function. Observe that $\varphi: \mathbb{R}^{m \times n} \rightarrow \overline{\mathbb{R}}=\mathbb{R} \cup\{+\infty\}$ is polyconvex and that

$$
\left\{\begin{array}{c}
\varphi(x) \leq 0, \text { if } x \in K \\
\varphi(x)=0, \text { if } x \in K_{e x t}^{p} .
\end{array}\right.
$$

Indeed the inequality is clear since in $K$ the function $f$ is finite and, by definition, $P f$ is always not larger than $f$. We now show that

$$
\varphi(x)=0 \Leftrightarrow x \in K_{\text {ext }}^{p} .
$$

Note that if $x \in K$ then

$$
\varphi(x)=|x|^{2}+\inf _{x_{i} \in K}\left\{\begin{array}{c}
-\sum_{i=1}^{\tau(m, n)+1} t_{i}\left|x_{i}\right|^{2}: T(x)=\sum_{i=1}^{\tau+1} t_{i} T\left(x_{i}\right) \\
t_{i} \geq 0 \text { with } \sum_{i=1}^{\tau+1} t_{i}=1
\end{array}\right\} .
$$

Therefore if $x \in K_{e x t}^{p}$, we deduce, by definition, that in the infimum the only admissible $x_{i}$ are $x_{i}=x$; and hence we have $\varphi(x)=0$. We now show the converse implication, i.e. $\varphi(x)=0 \Rightarrow x \in K_{\text {ext }}^{p}$. From the above representation formula we obtain, since $\varphi(x)=0$ and $x \in K$, that

$$
|x|^{2}=\sup _{x_{i} \in K}\left\{\sum_{i=1}^{\tau(m, n)+1} t_{i}\left|x_{i}\right|^{2}: T(x)=\sum_{i=1}^{\tau+1} t_{i} T\left(x_{i}\right)\right\} .
$$

Combining the above with the convexity of the function $x \rightarrow|x|^{2}$ we get that

$$
|x|^{2} \geq \sum_{i=1}^{\tau(m, n)+1} t_{i}\left|x_{i}\right|^{2} \geq\left|\sum_{i=1}^{\tau(m, n)+1} t_{i} x_{i}\right|^{2}=|x|^{2} ;
$$

the strict convexity of $x \rightarrow|x|^{2}$ implies then that $x_{i}=x$. Thus $x \in$ $K_{e x t}^{p}$. 
We now have the following version of Minkowski theorem.

Theorem 11.7. Let $E \subset \mathbb{R}^{m \times n}$ be a non empty compact set. Let $E_{\text {ext }}^{p}$ be the extreme points in the polyconvex sense of Pco E, then

$$
\text { Pco } E=\operatorname{Pco} E_{\text {ext }}^{p} \text {. }
$$

Proof. We adapt here an idea of Zhang [18].

Step 1 : We first prove that if $K$ is a compact and polyconvex set then it has at least one extreme point in the polyconvex sense, i.e. $K_{e x t}^{p} \neq \emptyset$. Let co $K$ be the convex hull of $K$, which is a compact and convex set. It is a well established fact in convex analysis that co $K$ has then at least one extreme point (in the convex sense). Since, by definition, any extreme point (in the convex sense) is an extreme point in the polyconvex sense, we deduce the result.

Step 2 : We next let

$$
K=\mathrm{Pco} E ; L=\mathrm{Pco} E_{e x t}^{p} .
$$

The only non trivial inclusion is $K \subset L$. We then define

$$
\left\{\begin{array}{c}
g(X)=\left\{\begin{array}{c}
\operatorname{dist}(X ; L), \text { if } X \in K \\
+\infty, \text { otherwise }
\end{array}\right. \\
f(X)=P g(X) \geq 0
\end{array}\right.
$$

We could also have taken, if we want a function that is finite everywhere,

$$
\left\{\begin{array}{c}
g(X)=[\operatorname{dist}(X ; L)]^{(m \wedge n)+1} \\
f(X)=P g(X) \geq 0
\end{array}\right.
$$

Observe that

$$
L=\{X \in K: f(X)=0\}
$$

(this follows from the polyconvexity of $L$ ). Let

$$
a=\max \{f(X): X \in K\} \geq 0 .
$$

We will show that $a=0$ which by (15) implies $K \subset L$ as claimed. Let

$$
\left\{\begin{array}{c}
E_{a}=\{X \in K: f(X)=a\} \neq \emptyset \\
\widetilde{K}=\operatorname{Pco} E_{a} \subset K .
\end{array}\right.
$$

Since $f$ is polyconvex and non negative we get

$$
\widetilde{K} \subset\{X \in K: 0 \leq f(X) \leq a\} .
$$

It follows from Step 1 that

$$
\emptyset \neq \widetilde{K}_{e x t}^{p} \subset E_{a} .
$$

Assume for a moment that we can show

$$
\emptyset \neq \widetilde{K}_{e x t}^{p} \subset E_{e x t}^{p}
$$


then combining (19), (20) and the fact that

$$
E_{\text {ext }}^{p} \subset L=\{X \in K: f(X)=0\}
$$

we would then deduce that

$$
\emptyset \neq \widetilde{K}_{e x t}^{p} \subset E_{a} \cap L .
$$

This implies at once that $a=0$ and hence $K \subset L$ as claimed.

So it only remains to show (20). We thus let $\xi \in \widetilde{K}_{e x t}^{p}$ be such that

$$
\left\{\begin{array}{c}
T(\xi)=\sum_{i=1}^{I} t_{i} T\left(\xi_{i}\right) \\
t_{i}>0 \text { with } \sum_{i=1}^{I} t_{i}=1, \xi_{i} \in K
\end{array}\right.
$$

and we wish to show that

$$
\xi_{i}=\xi, \forall i=1, \ldots, I
$$

which implies that $\xi \in E_{e x t}^{p}$ and hence (20). Ordering differently the $\xi_{i} \in K$, if necessary, we have

$$
\begin{aligned}
\xi_{i} & \in E_{a}\left(\Leftrightarrow f\left(\xi_{i}\right)=a\right) \Leftrightarrow i=1, \ldots, I_{1} \\
\xi_{i} & \in K \backslash E_{a}\left(\Rightarrow f\left(\xi_{i}\right)<a\right) \Leftrightarrow i=I_{1}+1, \ldots, I .
\end{aligned}
$$

We first show that $I_{1}=I$. If this were not the case we would have from (19) and the polyconvexity of $f$ that

$$
a=f(\xi) \leq \sum_{i=1}^{I} t_{i} f\left(\xi_{i}\right)=a \sum_{i=1}^{I_{1}} t_{i}+\sum_{i=I_{1}+1}^{I} t_{i} f\left(\xi_{i}\right)<a
$$

which is absurd, thus $I_{1}=I$. However since $\xi \in \widetilde{K}_{\text {ext }}^{p}$ (where $\widetilde{K}=$ Pco $\left.E_{a}\right)$ we therefore deduce that $(21)$ holds and hence $\xi \in E_{e x t}^{p}$ which is the claimed inclusion (20).

\section{Appendix: Singular values}

We recall here the definition and some properties of singular values of matrices (c.f. Horn-Johnson [11] page 152 and [6] page 171). First we write any $A \in \mathbb{R}^{m \times n}$ as

$$
A=\left(\begin{array}{lll}
a_{1}^{1} & \cdots & a_{n}^{1} \\
\vdots & & \vdots \\
a_{1}^{m} & \cdots & a_{n}^{m}
\end{array}\right)=\left(\begin{array}{l}
a^{1} \\
\vdots \\
a^{m}
\end{array}\right)=\left(a_{1}, \ldots, a_{n}\right)
$$

We start with the following 
Definition 12.1. We denote by $O(m, n)$ the set of orthogonal matrices $R \in \mathbb{R}^{m \times n}$, i.e.

$$
R^{t} R=I_{n \times n}
$$

where $I_{n \times n}$ denotes the identity matrix in $\mathbb{R}^{n \times n}$. When $m=n$, we write $O(n)=O(n, n)$.

Remark 12.2. If $m \neq n$, then in general

$$
R^{t} R=I_{n \times n} \nRightarrow R R^{t}=I_{m \times m}
$$

(i.e. $\left.R \in O(m, n) \nRightarrow R^{t} \in O(n, m)\right)$; while if $m=n$ then these two properties are equivalent (i.e. $R \in O(n) \Leftrightarrow R^{t} \in O(n)$ ).

We now give the definition of the singular values.

Definition 12.3. (1) Let $m \leq n$ and $A \in \mathbb{R}^{m \times n}$. The singular values of $A$, denoted by $0 \leq \lambda_{1}(A) \leq \ldots \leq \lambda_{m}(A)$, are defined to be the square root of the eigenvalues of the symmetric and positive semidefinite matrix $A A^{t} \in \mathbb{R}^{m \times m}$.

(2) Let $m \geq n$ and $A \in \mathbb{R}^{m \times n}$. The singular values of $A$, denoted by $0 \leq \lambda_{1}(A) \leq \ldots \leq \lambda_{n}(A)$, are defined to be the square root of the eigenvalues of the symmetric and positive semidefinite matrix $A^{t} A \in$ $\mathbb{R}^{n \times n}$.

The following theorem is the standard decomposition theorem (c.f. Horn-Johnson [10] Lemma 7.3.1).

Theorem 12.4. (1) Let $m \leq n, A \in \mathbb{R}^{m \times n}$ and $0 \leq \lambda_{1}(A) \leq \ldots \leq$ $\lambda_{m}(A)$ be its singular values then there exists $R \in O(m)$ such that

$$
R A=\widetilde{A}=\left(\begin{array}{l}
\widetilde{a}^{1} \\
\vdots \\
\widetilde{a}^{m}
\end{array}\right) \text {, with }\left\langle\widetilde{a}^{i} ; \widetilde{a}^{j}\right\rangle=\left|\widetilde{a}^{i}\right|\left|\widetilde{a}^{j}\right| \delta^{i j}, \lambda_{i}(A)=\left|\widetilde{a}^{i}\right| \text {. }
$$

Furthermore there exists $Q \in O(n, m) \subset \mathbb{R}^{n \times m}$ (i.e. $Q^{t} Q=I_{m \times m}$ ) such that

$$
R A Q=\left(\begin{array}{lll}
\lambda_{1}(A) & \cdots & 0 \\
\vdots & \ddots & \vdots \\
0 & \cdots & \lambda_{m}(A)
\end{array}\right)
$$

(2) Let $m \geq n, A \in \mathbb{R}^{m \times n}$ and $0 \leq \lambda_{1}(A) \leq \ldots \leq \lambda_{n}(A)$ be its singular values then there exists $R \in O(n)$ such that

$$
A R=\widetilde{A}=\left(\widetilde{a}_{1}, \ldots, \widetilde{a}_{n}\right) \text {, with }\left\langle\widetilde{a}_{i} ; \widetilde{a}_{j}\right\rangle=\left|\widetilde{a}_{i}\right|\left|\widetilde{a}_{j}\right| \delta_{i j}, \lambda_{i}(A)=\left|\widetilde{a}_{i}\right| .
$$


Furthermore there exists $Q \in \mathbb{R}^{n \times m}$ with $Q Q^{t}=I_{n \times n}$ (i.e. $Q^{t} \in$ $O(m, n))$ such that

$$
Q A R=\left(\begin{array}{lll}
\lambda_{1}(A) & \cdots & 0 \\
\vdots & \ddots & \vdots \\
0 & \cdots & \lambda_{n}(A)
\end{array}\right)
$$

\section{ACKNOWLEDGMENTS}

This research has been partially financed by Fonds National Suisse (21-50472.97). We would like to thank A. DeSimone, G. Dolzmann and P. Marcellini for intersting discussions.

\section{REFERENCES}

[1] Bousselsal M. and Le Dret H: Remarks on the quasiconvex envelope of some functions depending on quadratic forms; preprint (1999).

[2] Coddington E.A. and Levinson N.: Theory of ordinary differential equations; MacGraw-Hill, New York (1955).

[3] Dacorogna B.: Direct methods in the calculus of variations; Springer, Berlin (1989).

[4] Dacorogna B. and Marcellini P.: Existence of minimizers for non quasiconvex integrals; Archive Rational Mech. Anal. 131 (1995), 359-399.

[5] Dacorogna B. and Marcellini P.: Sur le problème de Cauchy-Dirichlet pour les systèmes d'équations non linéaires du premier ordre; C. R. Acad. Sci. Paris Ser. I Math. 323 (1996), 599-602.

[6] Dacorogna B. and Marcellini P.: Implicit partial differential equations; Birkhäuser, Boston (1999).

[7] Dacorogna B., Marcellini P. and Tanteri C.: Equations de type implicite avec contraintes; C. R. Acad. Sci. Paris Sér. I Math. 330 (2000), 271-274.

[8] Dacorogna B. and Tanteri C.: On the different convex hulls of sets involving singular values; Proc. Royal Soc. Edinburgh 128 A (1998), 1261-1280.

[9] DeSimone A. and Dolzmann G.: Material instabilities in nematic elastomers; Phys. D 136 (2000) no. 1-2, 175-191.

[10] Horn R.A. and Johnson C.A.: Matrix Analysis; Cambridge University Press (1985).

[11] Horn R.A. and Johnson C.A.: Topics in Matrix Analysis; Cambridge University Press (1991).

[12] Magnanini R. and Talenti G.: On complex valued solutions to a 2D eikonal equation. Part one, qualitative properties; edited by G.Q. Chen and E. DiBenedetto in American Mathematical Society, Contemporary Mathematics Series, Volume 238 (1999), 203-229.

[13] Müller S. and Sverak V.: Attainment results for the two-well problem by convex integration; ed. Jost J., International Press, (1996), 239-251.

[14] Müller S. and Sverak V.: Convex integration with constraints and applications to phase transitions and partial differential equations; J. Eur. Math.Soc. 1 (1999), 393-422. 
[15] Pianigiani G.: Differential inclusions. The Baire category method; in Methods of nonconvex analysis, edited by A. Cellina, Lecture Notes in Math., Springer, Berlin (1990), 104-136.

[16] Rockafellar R.T.: Convex Analysis; Princeton University Press, (1970).

[17] Tartar L.: Estimations fines des coefficients homogénéisés; in Ennio De Giorgi colloquium, edited by Krée P.; Research Notes in Math. 125, Pitman (1985), 168-187.

[18] Zhang K.: On the structure of quasiconvex hulls; Annales Institut Henri Poincaré, Analyse Non Linéaire 15 (1998), 663-686. 